\title{
How CMB and large-scale structure constrain chameleon interacting dark energy
}

\author{
Daniel Boriero, ${ }^{a}$ Subinoy Das, ${ }^{b}$ and Yvonne Y. Y. Wong ${ }^{c}$ \\ ${ }^{a}$ Fakultät für Physik, Universität Bielefeld, Universitätstr. 25, Bielefeld, Germany \\ ${ }^{b}$ Indian Institute of Astrophisics, Bangalore, 560034, India \\ ${ }^{c}$ School of Physics, The University of New South Wales, Sydney NSW 2052, Australia \\ E-mail: \\ boriero@physik.uni-bielefeld.de, subinoy@iiap.res.in, yvonne.y.wong@unsw.edu.au
}

\begin{abstract}
We explore a chameleon type of interacting dark matter-dark energy scenario in which a scalar field adiabatically traces the minimum of an effective potential sourced by the dark matter density. We discuss extensively the effect of this coupling on cosmological observables, especially the parameter degeneracies expected to arise between the model parameters and other cosmological parameters, and then test the model against observations of the cosmic microwave background (CMB) anisotropies and other cosmological probes. We find that the chameleon parameters $\alpha$ and $\beta$, which determine respectively the slope of the scalar field potential and the dark matter-dark energy coupling strength, can be constrained to $\alpha<0.17$ and $\beta<0.19$ using CMB data alone. The latter parameter in particular is constrained only by the late Integrated Sachs-Wolfe effect. Adding measurements of the local Hubble expansion rate $H_{0}$ tightens the bound on $\alpha$ by a factor of two, although this apparent improvement is arguably an artefact of the tension between the local measurement and the $H_{0}$ value inferred from Planck data in the minimal $\Lambda \mathrm{CDM}$ model. The same argument also precludes chameleon models from mimicking a dark radiation component, despite a passing similarity between the two scenarios in that they both delay the epoch of matter-radiation equality. Based on the derived parameter constraints, we discuss possible signatures of the model for ongoing and future large-scale structure surveys.
\end{abstract}




\section{Contents}

1 Introduction $\quad 1$

2 Chameleon model $\quad 2$

2.1 Background evolution 3

2.2 Attractor solution 4

2.3 Evolution of inhomogeneities 6

$\begin{array}{lll}3 & \text { Effects on cosmological observables } & 7\end{array}$

$\begin{array}{lll}3.1 & \text { CMB anisotropies } & 7\end{array}$

$\begin{array}{lll}3.2 & \text { Non-CMB observables } & 10\end{array}$

4 Cosmological data analysis $\quad 11$

$\begin{array}{lll}4.1 & \text { Data sets } & 11\end{array}$

$\begin{array}{lll}4.2 & \text { Parameter constraints } & 13\end{array}$

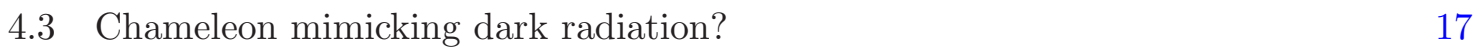

$\begin{array}{llr}5 & \text { Future tests } & 18\end{array}$

6 Conclusions $\quad 19$

\section{Introduction}

There is a growing body of evidence that the universe is currently undergoing a phase of accelerated expansion (e.g., [1-6]). This apparent acceleration is usually attributed to a dark energy with an equation of state $w \simeq-1$. The fundamental nature of dark energy, however, remains unknown. At present, a cosmological constant or vacuum energy with $w=-1$ appears to be simplest solution that can account for all available data. But dynamical scalar field models of dark energy [7-9] or $f(R)$ modified gravity models [10-12] remain viable, albeit highly constrained, possibilities.

Yet another interesting possibility are the interacting quintessence models [13]. Here, a scalar field responsible for driving the accelerated expansion interacts with dark matter and/or ordinary matter. ${ }^{1}$ In fact, in the absence of any symmetry forbidding the interaction, it is quite natural to expect such couplings $[17,18]$. An immediate consequence, however, is that the matter fields will perceive an additional long-range force on super-Mpc scales; in the case the scalar field couples to baryonic matter, any such "fifth-force" effect that may have trickled down to the sub-Mpc scales is automatically subject to solar system constraints [19, $20]$ as well as bounds from laboratory test of gravity [21, 22].

One possible way to avoid these local constraints is the chameleon mechanism. The basic idea is that because the scalar field interacts with matter, its mass is a function of the local matter density. In those chameleon dark energy models wherein the scalar field couples universally to all forms of matter, the high baryonic matter density on solar system and

\footnotetext{
${ }^{1}$ It has been shown that any $f(R)$ model can be mapped to an interacting quintessence model by a suitable conformal transformation of the metric to the Einstein frame. In a sense, both $f(R)$ and interacting quintessence are scalar-tensor modifications of gravity that introduce a scalar fifth force [14-16].
} 
laboratory scales causes the scalar field to become heavy, thereby hiding the fifth force from local tests. This screening mechanism switches off on matter-thin super-Galactic scales, and ideally the fifth force should invert its behaviour so as to drive the accelerated expansion of the universe [23-27]. However, a recent work [28] has shown that the range of the chameleon force in universally-coupled models is necessarily restricted to below the $\sim 1 \mathrm{Mpc}$ scale, so that on its own the chameleon field is in fact unable to account for the observed accelerated expansion. This constraint can nonetheless be circumvented if the scalar field interacts only with non-baryonic matter, and the chameleon mechanism as discussed above remains viable.

In this work, we consider one such interacting dark matter-dark energy (DM-DE) model proposed in [29], in which the scalar field adiabatically tracks the minimum of the effective potential starting from deep in the radiation-dominated era up to the present epoch. This model is very simple in that the coupling between the dark matter spinor $\psi$ and the scalar field $\phi$ is of the Yukawa type, $f(\phi) \psi \bar{\psi}$, and the coupling function is chosen to be a positive exponential suppressed by the Planck scale, i.e., $f(\phi)=e^{\beta \phi / M_{\mathrm{P} 1}}$, which is very common in the Einstein frame and emerges from many string theory models of compactification [30]; the selfinteraction potential for $\phi$ takes the runaway form $V(\phi) \sim \phi^{-\alpha}$. Previously, a naïve estimate has put an upper limit on $\alpha, \alpha \leq 0.2$, while $\beta$, which controls the strength of the fifth force, was found to be practically unconstrained [29]. In the present work, we wish to confront this model with the most recent cosmological data, especially measurements of the cosmic microwave background (CMB) temperature anisotropies by the ESA Planck mission [5], and reexamine the model's viability.

Our second motivation comes from recent hints of a possible excess of radiation energy density during the CMB decoupling epoch from measurements of the CMB damping tail by the Atacama Cosmology Telescope (ACT) [31] and the South Pole Telescope (SPT) [32], as well as from the combined analysis of Planck data and the Hubble parameter inferred in the local neighbourhood $[5,6]$. Conventional explanations include a thermalised populatioin of eV-mass sterile neutrinos (e.g., [33, 34]) or other light particles (e.g., [35]), relativistic decay products of heavy particles (e.g., [36-38]), as well as early dark energy [39]. However, ultimately, what the CMB anisotropies probe are the effects of the energy content on the evolution of the photon perturbations around the decoupling epoch [40,41]. To this end, chameleon models, in which the dark matter-dark energy interaction endows the dark matter component with a nonstandard time evolution that tends to delay the epoch of matterradiation equality, may very well mimic the phenomenology of a radiation excess.

The plan of the paper is as follows. We begin in section 2 with a brief review of the chameleon model, and present the relevant equations of motion. In section 3 we discuss the effects of the chameleon model on cosmological observables, and the parameter degeneracies expected to arise between the model parameters $\alpha$ and $\beta$ and other cosmological parameters. We perform a fit of the model to cosmological data in section 4 , and discuss the implications for the chameleon model parameters as well as the possibility of chameleon dark energy mimicking dark radiation. Using the results from section 4, we identify in section 5 possible observational signatures for future cosmological probes. We state our conclusions in section 6 .

\section{Chameleon model}

We briefly review the chameleon model of [29] in this section, and present the relevant equations that determine the background evolution, the initial conditions, and as well as the evolution of the dark energy and dark matter perturbations. These equations will later 
be embedded into the Boltzmann code CAMB [42], in order to analyse the impact of the chameleon mechanism on cosmological observables such as the CMB anisotropies and the large-scale structure distribution.

\subsection{Background evolution}

Following [25, 43], we take the Einstein frame as the physical frame in which dark energy interacts with dark matter fields through a $\phi$-dependent conformally rescaled Jordan frame metric $g_{\mu \nu}^{(i)}$, whose generic form is given by

$$
g_{\mu \nu}^{(i)}=e^{2 \beta_{i} \phi / M_{\mathrm{Pl}}} g_{\mu \nu}
$$

where $\beta_{i}$ are dimensionless coupling constants, and $M_{\mathrm{P} 1}$ is the reduced Planck mass. The matter spinor fields $\psi^{(i)}$ are understood to follow the geodesics of $g_{\mu \nu}^{(i)}$, while the original $g_{\mu \nu}=\operatorname{diag}\left(-1, a^{2}, a^{2}, a^{2}\right)$ is the flat Friedmann-Lemaittre-Robertson-Walker (FLRW) metric in the Einstein frame with scale factor $a$. The action takes the form

$$
S=\int d^{4} x\left\{\sqrt{-g}\left[\frac{M_{\mathrm{Pl}}^{2}}{2} \mathcal{R}-\frac{(\partial \phi)^{2}}{2}-V(\phi)\right]+\mathcal{L}_{m}\left(\psi^{(i)}, g_{\mu \nu}^{(i)}\right)\right\},
$$

where $\mathcal{R}$ is the Ricci scalar, $\mathcal{L}_{m}$ the Lagrangian density for the matter fields, and we have also added the kinetic and potential terms for the scalar field $\phi$. Varying the action with respect to $\phi$, we obtain an equation of motion for the scalar field:

$$
\ddot{\phi}+3 H \dot{\phi}=-V_{, \phi}(\phi)+\sum_{i} \frac{\beta_{i}}{M_{\mathrm{Pl}}} e^{4 \beta_{i} \phi / M_{\mathrm{Pl}}} g_{(i)}^{\mu \nu} T_{\mu \nu}^{(i)}
$$

where an overhead "." denotes a derivative with respect to the cosmic time $t, H \equiv \dot{a} / a$ is the Hubble expansion rate, $T_{\mu \nu}^{(i)} \equiv\left(-2 / \sqrt{-g^{(i)}}\right) \delta \mathcal{L}_{m} / \delta g_{(i)}^{\mu \nu}$ the Jordan frame energy-momentum tensor of the $i$ th matter field, and we have assumed that the scalar field is homogeneous and isotropic. Limiting the coupling to the dark sector, equation (2.3) reduces to

$$
\ddot{\phi}+3 H \dot{\phi}=-V_{, \phi}(\phi)-\frac{\beta}{M_{\mathrm{Pl}}} e^{4 \beta \phi / M_{\mathrm{Pl}}} \tilde{\rho}_{\mathrm{DM}},
$$

where $\tilde{\rho}_{\mathrm{DM}} \equiv-g_{\mu \nu}^{(\mathrm{DM})} T_{(\mathrm{DM})}^{\mu \nu}$ is the Jordan frame mean dark matter energy density.

To determine the evolution of $\tilde{\rho}_{\mathrm{DM}}$, conservation of energy-momentum $T_{\nu ; \mu}^{\mu(\mathrm{DM})}=0$ in the Jordan frame gives

$$
\dot{\tilde{\rho}}_{\mathrm{DM}}+3\left(H+\frac{\beta}{M_{\mathrm{Pl}}} \dot{\phi}\right) \tilde{\rho}_{\mathrm{DM}}=0
$$

which has the solution

$$
\tilde{\rho}_{\mathrm{DM}}=\frac{\tilde{\rho}_{\mathrm{DM}}^{(0)}}{a^{3}} e^{-3 \beta\left(\phi-\phi_{0}\right) / M_{\mathrm{Pl}}}=\frac{\rho_{\mathrm{DM}}^{(0)}}{a^{3}} e^{-\beta\left(3 \phi+\phi_{0}\right) / M_{\mathrm{Pl}}},
$$

where $\phi_{0} \equiv \phi(a=1)$, and $\rho_{\mathrm{DM}}^{(0)} \equiv \tilde{\rho}_{\mathrm{DM}}^{(0)} e^{4 \beta \phi_{0} / M_{\mathrm{Pl}}}$ is identified as the present-day physical dark matter density in the Einstein frame. Substituting equation (2.6) into (2.4), we see immediately that the scalar field dynamics in this model is controlled by an effective potential

$$
V_{\mathrm{eff}}(\phi)=V(\phi)+\frac{\rho_{\mathrm{DM}}^{(0)}}{a^{3}} e^{\beta\left(\phi-\phi_{0}\right) / M_{\mathrm{Pl}}},
$$


where, for this work, we adopt a simple power law runaway potential,

$$
V(\phi)=M_{\phi}^{4}\left(\frac{M_{\mathrm{P} 1}}{\phi}\right)^{\alpha}
$$

with $\alpha$ a positive constant that characterises the scalar field's self-interaction, and $M_{\phi}$ the scalar field mass. This form of potential is common in many string compactification models [30], and has the property that the attractor solution possesses a growing DM-DE coupling. In the limit $\alpha=0$, the model reduces to the case of a cosmological constant.

The Friedmann equation for the full system, including other standard forms of energy densities, reads

$$
H^{2}(t)=\left(\frac{\dot{a}}{a}\right)^{2}=\frac{1}{3 M_{\mathrm{Pl}}^{2}}\left[\frac{\rho_{\gamma}^{(0)}}{a^{4}}+\frac{\rho_{\nu}^{(0)}}{a^{4}}+\frac{\rho_{\mathrm{B}}^{(0)}}{a^{3}}+\frac{\rho_{\mathrm{DM}}^{(0)}}{a^{3}} e^{\beta\left(\phi-\phi_{0}\right) / M_{\mathrm{Pl}}}+\frac{\dot{\phi}^{2}}{2}+V(\phi)\right],
$$

where $\rho_{\gamma}^{(0)}, \rho_{\nu}^{(0)}$ and $\rho_{\mathrm{B}}^{(0)}$ denote the present-day energy densities of photons, massless neutrinos and baryons, respectively. Again, for a vanishing coupling $\beta$ and a constant potential $V(\phi)$, equation (2.9) reduces to the standard $\Lambda$ CDM equation. Observe that the DM-DE interaction induces a nonstandard time evolution for the dark matter density, i.e.,

$$
\rho_{\mathrm{DM}}=\frac{\rho_{\mathrm{DM}}^{(0)}}{a^{3}} e^{\beta\left(\phi-\phi_{0}\right) / M_{\mathrm{P} 1}} .
$$

Furthermore, while the equation of state for the scalar field alone is the canonical one, namely,

$$
w_{\phi} \equiv \frac{P_{\phi}}{\rho_{\phi}}=\frac{\dot{\phi}^{2} / 2-V(\phi)}{\dot{\phi}^{2} / 2+V(\phi)},
$$

where $\rho_{\phi}$ and $P_{\phi}$ are, respectively, the scalar field's energy density and pressure, taking into account the nonstandard dark matter density (2.10), the effective dark energy density (as far as the background expansion rate $H(t)$ is concerned) is in fact

$$
\rho_{\mathrm{DE}}=\rho_{\phi}+\frac{\rho_{\mathrm{DM}}^{(0)}}{a^{3}}\left(e^{\beta\left(\phi-\phi_{0}\right) / M_{\mathrm{P} 1}}-1\right) \equiv(1-x) \rho_{\phi},
$$

which has an effective equation of state

$$
w_{\text {eff }}=w_{\phi} \times(1-x)^{-1} .
$$

Where the coupling increases with time, such as in our case, $x \geqslant 0$ holds, thereby driving $w_{\text {eff }}$ to a value below -1 , and hence leading the dark energy to exhibit an apparent phantom behaviour.

\subsection{Attractor solution}

The attractor solution for chameleon models and its stability have been studied previously in $[25,43,44]$. It is set by the minimum of the effective potential, i.e., $V_{\text {eff }}^{, \phi}\left(\phi_{\min }\right)=0$, and adiabatic evolution simply stipulates that $\phi(t)$ traces the minimum at all times. For the potential (2.8), this means

$$
f[\phi(t)]=\left(\frac{\phi_{0}}{\phi(t)}\right)^{\alpha+1}-\frac{e^{\beta\left(\phi(t)-\phi_{0}\right) / M_{\mathrm{P} 1}}}{a^{3}(t)} \rightarrow 0
$$



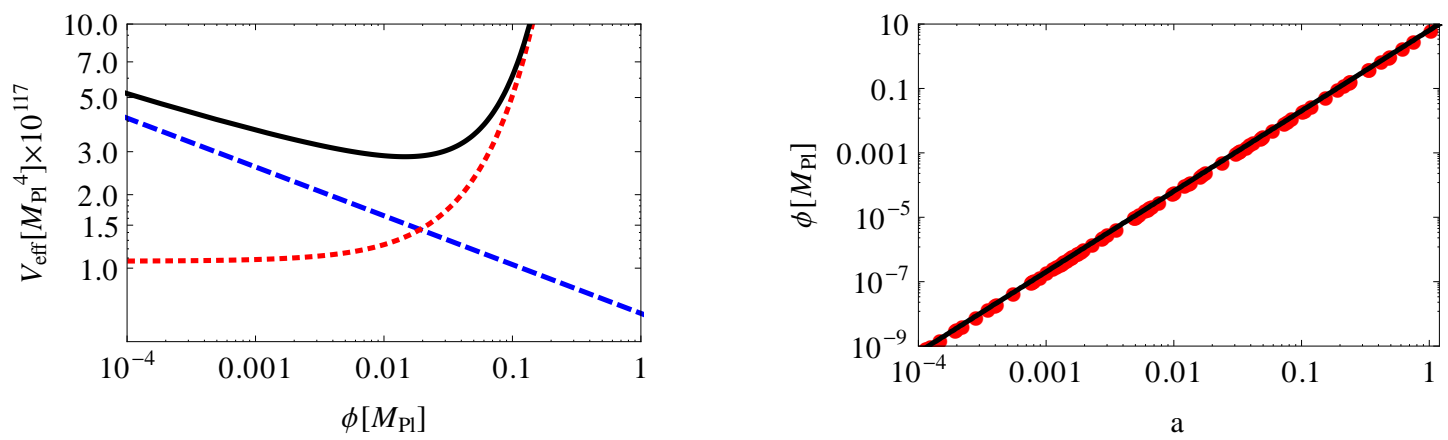

Figure 1. Left: The effective potential $V_{\text {eff }}$ (solid black) for the model parameters $\left(\alpha, \beta, \omega_{\mathrm{DM}}, h\right)=$ $(0.2,0.1,0.13,0.7)$. It is a composite of the original potential $V(\phi)$ (dashed blue) and the coupling with dark matter (dotted red). Right: The corresponding $\phi$ field as a function of the scale factor $a$, obtained from numerically solving equation (2.4) (dotted red), and from the analytical solution (2.14) (solid black).

which can be solved using a simple bisection algorithm once the scalar field's present-day value $\phi_{0}$ has been determined. The latter can be achieved by demanding that the field's present-day potential energy dominates over its kinetic energy, so that $\rho_{\phi}^{(0)} \approx V\left(\phi_{0}\right)$. Then from $V_{\text {eff }}^{, \phi}\left(\phi_{\min }\right)=0$ we find

$$
\frac{\phi_{0}}{M_{\mathrm{Pl}}} \approx \frac{\alpha}{\beta} \times \frac{\rho_{\phi}^{(0)}}{\rho_{\mathrm{DM}}^{(0)}}=\frac{\alpha}{\beta}\left(\frac{h^{2}}{\omega_{\mathrm{DM}}}-1\right),
$$

where the last equality in terms of the reduced density parameter $\omega_{\mathrm{DM}}$ and Hubble parameter $h$ follows from the assumption of a flat spatial geometry. An estimate for the mass scale of $\phi$ field,

$$
M_{\phi} \approx\left[\rho_{\phi}^{(0)}\left(\frac{\phi_{0}}{M_{\mathrm{Pl}}}\right)^{\alpha}\right]^{1 / 4},
$$

also follows immediately. Thus, once $\omega_{\mathrm{DM}}$ an $h$ have been specified, it is possible to uniquely parameterise an adiabatically evolving chameleon model with only two parameters $\alpha$ and $\beta$ in a spatially flat universe.

Figure 1 shows the effective potential $V_{\text {eff }}(\phi)$ for choice of parameters $\left(\alpha, \beta, \omega_{\mathrm{DM}}, h\right)=$ $(0.2,0.1,0.13,0.7)$, and the corresponding evolution of $\phi$ - the numerical solution of equation (2.4) as well as the analytical solution (2.14) - as a function of the scale factor $a$. Note that we have chosen the initial value of the scalar field $\phi^{\text {in }}$ to be in the undershooting regime, i.e., $0<\phi^{\text {in }}<\phi_{\min }^{\text {in }}$. This ensures the satisfaction of the slow-roll conditions,

$$
V_{, \phi \phi}^{1 / 2} \gg H, \quad \dot{\phi}^{2} \ll 2 V(\phi),
$$

at all times, and consequently the stability of the linear density perturbations [44].

An immediate phenomenological consequence of the attractor solution is that the effective dark energy equation of state $w_{\text {eff }}$ as defined in equation (2.13), and consequently the cosmological background evolution, can have no dependence on the coupling parameter $\beta$. This is easy to see by first defining $\tilde{\phi} \equiv \beta \phi / M_{\mathrm{Pl}}$. Then the present-day field value is given by $\tilde{\phi}_{0} \approx \alpha\left(h^{2} / \omega_{\mathrm{DM}}-1\right)$ according to equation (2.15). Substituting this into the attractor 
solution (2.14), we see straight away that $\tilde{\phi}(t)$ does not depend on $\beta$. It follows then that $w_{\text {eff }}$, whose modification due to the DM-DE coupling comes in through the exponential $e^{\tilde{\phi}-\tilde{\phi}_{0}}$, is also independent of $\beta$. To measure $\beta$ we need to consider its effects on the evolution of the inhomogeneities.

\subsection{Evolution of inhomogeneities}

The presence of a DM-DE coupling likewise modifies the evolution of the inhomogeneities in the universe. Working in the synchronous gauge whose line element is

$$
d s^{2}=-d t^{2}+a^{2}(t)\left(\delta_{i j}+\tilde{h}_{i j}\right) d x^{i} d x^{j},
$$

the equations of motion for the dark matter density contrast $\delta_{\mathrm{DM}}(k, t)$ and velocity divergence $\theta_{\mathrm{DM}}(k, t)$ for Fourier mode $k$ are (e.g., [44])

$$
\begin{aligned}
& \dot{\delta}_{\mathrm{DM}}=-\left(\frac{\theta_{\mathrm{DM}}}{a}+\frac{\dot{\tilde{h}}}{2}\right)+\frac{\beta}{M_{\mathrm{Pl}}} \delta \dot{\phi}, \\
& \dot{\theta}_{\mathrm{DM}}=-H \theta_{\mathrm{DM}}+\frac{\beta}{M_{\mathrm{Pl}}}\left(\frac{k^{2}}{a} \delta \phi-\dot{\phi} \theta_{\mathrm{DM}}\right),
\end{aligned}
$$

and similarly for the scalar field perturbations,

$$
\ddot{\delta \phi}+3 H \dot{\delta \phi}+\left(\frac{k^{2}}{a^{2}}+V_{, \phi \phi}\right) \delta \phi+\frac{1}{2} \dot{\tilde{h}} \dot{\phi}=-\frac{\beta}{M_{\mathrm{Pl}}} \rho_{\mathrm{DM}} \delta_{\mathrm{DM}},
$$

where $\tilde{h} \equiv \tilde{h}_{i}^{i}$ is the trace of metric perturbation $\tilde{h}_{i j}$. Note that in non-interacting models the dark matter velocity divergence $\theta_{\mathrm{DM}}$ is exactly vanishing at all times because the synchronous coordinates are defined such that initially $\dot{\theta}_{\mathrm{DM}}^{\text {in }}=\theta_{\mathrm{DM}}^{\text {in }}=0$, and there is no source term to perturb $\theta_{\mathrm{DM}}$ away from zero. In chameleon models, however, the DM-DE coupling engenders a source term proportional to the scalar field perturbation $\delta \phi$, so that it becomes necessary to track the evolution of $\theta_{\mathrm{DM}}$ as well.

The equation of motion for the metric perturbation $\tilde{h}$, obtained from the perturbed Einstein equation, likewise needs to be modified according to

$$
\begin{aligned}
k^{2} \eta-\frac{1}{2} a^{2} H \dot{\tilde{h}} & =-\frac{a^{2}}{2 M_{\mathrm{Pl}}^{2}}\left(\sum_{i=\gamma, \nu, \mathrm{B}, \mathrm{DM}} \rho_{i} \delta_{i}+\delta \rho_{\phi}\right), \\
k^{2} \dot{\eta} & =\frac{a}{2 M_{\mathrm{Pl}}^{2}}\left(\sum_{i=\gamma, \nu, \mathrm{B}, \mathrm{DM}}\left(\rho_{i}+P_{i}\right) \theta_{i}+a k^{2} \dot{\phi} \delta \phi\right), \\
\ddot{\tilde{h}}+3 H \dot{\tilde{h}}-2 \frac{k^{2}}{a^{2}} \eta & =-\frac{3}{M_{\mathrm{Pl}}^{2}}\left(\sum_{i=\gamma, \nu, \mathrm{B}, \mathrm{DM}} \delta P_{i}+\dot{\phi} \delta \dot{\phi}-V_{, \phi} \delta \phi\right),
\end{aligned}
$$

where $\delta \rho_{\phi}=\dot{\phi} \delta \dot{\phi}+V_{, \phi} \delta \phi$, and we observe that perturbations in the $\phi$ field do not contribute to the traceless part of the spatial stress tensor. Note that the evolution equations presented in this section are common to all scalar-field-based couple DM-DE models; the uniqueness of the chameleon model lies in the behaviour of the potential and the existence of an attractor solution. 
It is instructive to rewrite equation (2.19) as a second order differential equation for $\delta_{\mathrm{DM}}$,

$$
\ddot{\delta}_{\mathrm{DM}}+2 H \dot{\delta}_{\mathrm{DM}}+\frac{1}{2}(\ddot{\tilde{h}}+2 H \dot{\tilde{h}}) \approx \frac{\beta^{2}}{M_{\mathrm{Pl}}^{2}} \frac{\rho_{\mathrm{DM}} \delta_{\mathrm{DM}}}{1+a^{2} V_{, \phi \phi} / k^{2}},
$$

where we have ignored terms proportional to $\dot{\phi}$ because of the slow-roll conditions (2.17), and solved equation (2.20) for $\delta \phi$ assuming a steady state (i.e., $\ddot{\delta \phi}=\dot{\delta \phi}=0$ ). While the term dependent on the metric perturbation $\tilde{h}$ arises from standard Einsteinian gravity and can be easily constructed from equations (2.21) and (2.23), the additional term proportional to $\beta^{2}$ can be interpreted as a fifth force acting on the dark matter perturbations. The (comoving) range of this fifth force is determined by

$$
\lambda_{\mathrm{F}}(a) \equiv a^{-1} V_{, \phi \phi}^{-1 / 2},
$$

so that only those scales satisfying $k>\lambda_{\mathrm{F}}^{-1}$ will feel the force's effect. For potentials of the form (2.8), we find

$$
\lambda_{\mathrm{F}}(a)=a^{-1} \sqrt{\frac{\phi^{\alpha+2}}{\alpha(\alpha+1) M_{\phi}^{4} M_{\mathrm{Pl}}^{\alpha}}} \approx a^{2} H_{0}^{-1} \sqrt{\frac{\Omega_{\phi}}{\Omega_{\mathrm{DM}}^{2}} \frac{\alpha}{3 \beta^{2}}} e^{-\beta\left(\phi-\phi_{0}\right) / M_{\mathrm{P} 1}},
$$

where we have assumed in the last equality $\alpha \ll 1$. Thus, while $\lambda_{F}$ today is comparable to the present-day Hubble length, at early times the range of the force is strongly suppressed by the scale factor $a$. At the time of CMB decoupling, for example, equation (2.26) evaluates to

$$
\lambda_{\mathrm{F}}\left(a_{*}\right) \approx a_{*}^{3 / 2}\left(a_{*} H_{*}\right)-1 \sqrt{\frac{\Omega_{\phi}}{\Omega_{\mathrm{DM}}} \frac{\alpha}{3 \beta^{2}}} e^{-\beta\left(\phi-\phi_{0}\right) / M_{\mathrm{Pl}}},
$$

which shows that $\lambda_{\mathrm{F}}\left(a_{*} \sim 0.001\right)$ is no more than about $10^{-5}$ times the comoving Hubble length at $a_{*}$, and consequently completely out of the observable range of the CMB primary anisotropies $\left(>\mathcal{O}(0.1) \times\left(a_{*} H_{*}\right)^{-1}\right)$.

\section{Effects on cosmological observables}

We describe in this section the observational consequences of the chameleon model for the current generation of cosmological probes. In particular, we discuss the parameter degeneracies expected to arise between the $\alpha$ and $\beta$ and other cosmological parameters, and determine which combinations of cosmological observations would be capable of lifting these degeneracies. We defer the discussion of chameleon effects for future observations to section 5 .

\subsection{CMB anisotropies}

Because the DM-DE coupling causes the dark matter density to dilute more slowly than $a^{-3}$, for the same present-day $\rho_{\mathrm{DM}}^{(0)}$ value, the chameleon model has a lower dark matter density at high redshifts than does the standard $\Lambda$ CDM case. This has the effect of delaying the epoch of matter-radiation equality as per

$$
\begin{aligned}
1+z_{\mathrm{eq}} & \approx \frac{\omega_{\mathrm{DM}} e^{-\beta \phi_{0} / M_{\mathrm{P} 1}}+\omega_{\mathrm{B}}}{\omega_{\gamma}} \frac{1}{1+0.227 N_{\mathrm{eff}}} \\
& \approx \frac{\omega_{\mathrm{DM}} e^{-\alpha\left(h^{2} / \omega_{\mathrm{DM}}-1\right)}+\omega_{\mathrm{B}}}{\omega_{\gamma}} \frac{1}{1+0.227 N_{\mathrm{eff}}},
\end{aligned}
$$


where $N_{\text {eff }}=3.046$, and the second approximate equality follows from equation (2.15). Changing the epoch of equality has a profound impact on the odd acoustic peak ratios of the CMB temperature anisotropies, and with seven acoustic peaks now observed by Planck, $z_{\mathrm{eq}}$ and hence $\omega_{\mathrm{DM}} e^{-\alpha\left(h^{2} / \omega_{\mathrm{DM}}-1\right)}$ can be considered a well-constrained quantity. ${ }^{2}$

However, merely measuring $\omega_{\mathrm{DM}} e^{-\alpha\left(h^{2} / \omega_{\mathrm{DM}}-1\right)}$ is clearly not sufficient to determine all of the chameleon model parameters, because of the intricate dependence of the exponent on the Hubble parameter $h$, the potential's slope $\alpha$, as well as $\omega_{\mathrm{DM}}$ itself, let alone the missing $\beta$. Breaking the degeneracy requires that we measure three other parameter combinations; two of these come automatically from the CMB temperature anisotropies:

Angular sound horizon. The $\theta_{\mathrm{s}}=r_{\mathrm{s}}^{*} / D_{\mathrm{A}}^{*}$ parameter determines the positions of the CMB acoustic peaks. In the numerator, $r_{\mathrm{s}}^{*} \equiv r_{\mathrm{s}}\left(a_{*}\right)=\int_{0}^{t_{*}} d t c_{\mathrm{s}}(t) / a(t)$ is the comoving sound horizon at the time of $\mathrm{CMB}$ decoupling. For the chameleon model this evaluates, up to a constant factor, to

$$
r_{\mathrm{s}}^{*} \propto \sqrt{\frac{4}{3} \frac{a_{\mathrm{eq}}}{\left(\omega_{\mathrm{DM}} e^{-\alpha\left(h^{2} / \omega_{\mathrm{DM}}-1\right)}+\omega_{\mathrm{B}}\right) R\left(z_{\mathrm{eq}}\right)}} \ln \left[\frac{\sqrt{1+R\left(z_{*}\right)}+\sqrt{R\left(z_{*}\right)+R\left(z_{\mathrm{eq}}\right)}}{1+\sqrt{R\left(z_{\mathrm{eq}}\right)}}\right],
$$

where $R(z) \equiv(3 / 4)\left(\omega_{\mathrm{B}} / \omega_{\gamma}\right) a$. In the denominator, $D_{\mathrm{A}}^{*} \equiv D_{\mathrm{A}}\left(a_{*}\right)$ is the angular diameter distance to the last scattering surface, which in the chameleon model takes the approximate form

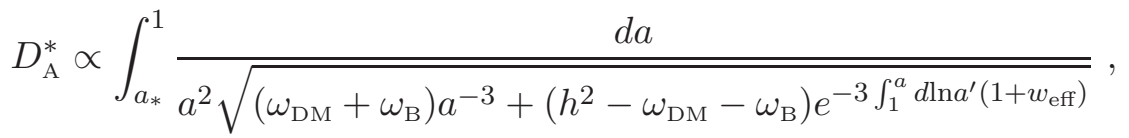

up to a constant factor. Measuring $z_{\text {eq }}$ and $\omega_{\mathrm{B}}$ from the peak ratios completely fixes $r_{\mathrm{s}}^{*}$, but not $D_{\mathrm{A}}^{*}$ which depends on a different combination of $\omega_{\mathrm{DM}}$ and $h$, as well as $\alpha$ through the effective dark energy equation of state $w_{\text {eff }}$ (as already discussed in section 2.2, $w_{\text {eff }}$ does not depend on $\beta)$. Therefore, $\theta_{\mathrm{s}}$ contributes towards breaking the $\left(\alpha, h, \omega_{\mathrm{DM}}\right)$-degeneracy.

Late Integrated Sachs-Wolfe (ISW) effect. The late ISW effect is primarily sensitive to the evolution of metric perturbations on those length scales that enter the Hubble horizon while the universe transitions from matter to dark energy domination at low redshifts, $z \lesssim 1$. The metric perturbations evolve according to equations (2.21) to (2.23) in the chameleon model, and have contributions from both the matter component and the scalar field.

The scalar field density perturbation $\delta \rho_{\phi}$ is given approximately by

$$
\delta \rho_{\phi} \approx V_{, \phi} \delta \phi \approx 3 \beta^{2} \Omega_{\mathrm{DM}}(t) \frac{a^{2} H^{2}(t)}{k^{2}} \frac{\rho_{\mathrm{DM}}}{1+a^{2} V_{, \phi \phi} / k^{2}} \delta_{\mathrm{DM}},
$$

where we have used $V_{, \phi}=-\left(\beta / M_{\mathrm{PI}}\right) \rho_{\mathrm{DM}}$, and assumed the steady-state solution of equation (2.20) for $\delta \phi$ (i.e., $\ddot{\delta \phi}=\dot{\delta \phi}=0$ ). Feeding this solution into equations (2.21) and (2.23) also allows us to rewrite the evolution equation (2.24) for the matter perturbations in the low-redshift universe as

$$
\ddot{\delta}_{\mathrm{DM}}+2 H \dot{\delta}_{\mathrm{DM}}=\frac{3}{2} H^{2} \Omega_{\mathrm{DM}}(t)\left[1+\frac{2 \beta^{2}}{1+a^{2} V_{, \phi \phi} / k^{2}}\left(1-3 \Omega_{\mathrm{DM}}(t) \frac{a^{2} H^{2}(t)}{k^{2}}\right)\right] \delta_{\mathrm{DM}},
$$

where we have neglected the baryon component for simplicity. Since according to equation (2.26) the range of the fifth force has recently attained Hubble length, we can establish

\footnotetext{
${ }^{2}$ The baryon density $\omega_{\mathrm{B}}$ is independently well constrained by the odd-to-even acoustic peak ratios.
} 

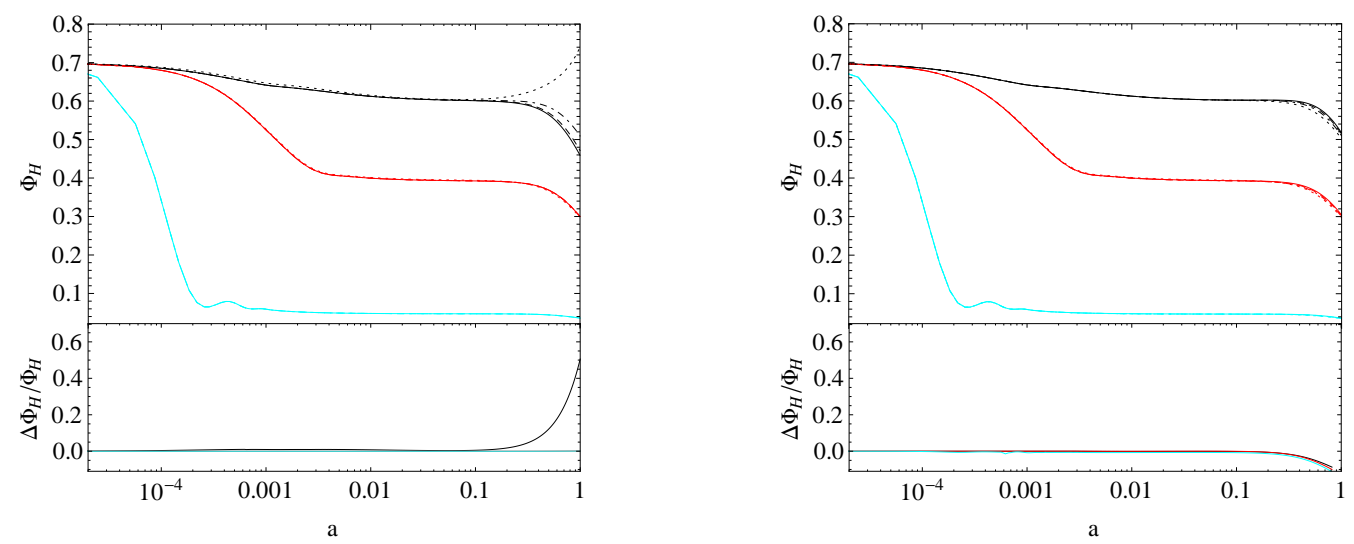

Figure 2. Bardeen potential $\Phi_{H}(k)$ as a function of the scale factor $a$ for $k=0.0001 h \mathrm{Mpc}^{-1}$ (black), $0.01 \mathrm{~h} \mathrm{Mpc}^{-1}$ (red), and $0.1 \mathrm{~h} \mathrm{Mpc}^{-1}$ (cyan). Left: Chameleon models with a fixed $\alpha=0.1$, and $\beta$ values of 0.01 (solid), 0.05 (dashed), 0.1 (dot-dashed) and 0.2 (dotted); the bottom portion shows the fractional difference $\Delta \Phi_{H} / \Phi_{H}$ between the $\beta=0.01$ and 0.2 cases. Right: Chameleon models with a fixed $\beta=0.1$, and $\alpha$ values of 0.01 (solid), 0.05 (dashed), 0.1 (dot-dashed) and 0.2 (dotted); the bottom portion shows the fractional difference between the $\alpha=0.01$ and 0.2 cases. In both panels all other cosmological parameters have been fixed to the $\Lambda$ CDM best-fit values from Planck +WP $+\mathrm{HST}$.

that $a^{2} V_{, \phi \phi} / k^{2} \lesssim 1$ for all observable scales at $z \lesssim 1$. It then follows from equation (3.4) that $\delta \rho_{\phi}$ is strongly dependent on the coupling parameter $\beta$, and its impact on the metric perturbations - both directly and indirectly through $\delta_{\mathrm{DM}}$-is greatest on scales close to the Hubble length due to its $a^{2} H^{2}(t) / k^{2}$ dependence. We illustrate this point in figure 2 with the gauge-invariant Bardeen potential,

$$
\Phi_{H} \equiv \frac{a^{2}}{2 M_{\mathrm{Pl}}^{2} k^{2}}\left\{\sum_{i=\gamma, \nu, \mathrm{B}, \mathrm{DM}}\left[\rho_{i} \delta_{i}+\frac{3 a H}{k^{2}}\left(\rho_{i}+P_{i}\right) \theta_{i}\right]+\delta \rho_{\phi}+3 a^{2} H \dot{\phi} \delta \phi\right\},
$$

as a function of the scale factor $a$ at $k=0.0001,0.01,0.1 h \mathrm{Mpc}^{-1}$ for various combinations of $\alpha$ and $\beta$.

A second interesting feature in equations (3.4) and (3.5) is that, while the low-redshift behaviour of $H(t)$ can be conveniently reparameterised in terms of an effective dark energy equation of state $w_{\text {eff }}$, the time-dependent reduced density parameter $\Omega_{\mathrm{DM}}(t)$, given in this case by

$$
\Omega_{\mathrm{DM}}(t) \equiv \frac{\rho_{\mathrm{DM}}^{(0)} a^{-3}}{\rho_{\mathrm{DM}}^{(0)} a^{-3}+\rho_{\phi} e^{-\beta\left(\phi-\phi_{0}\right) / M_{\mathrm{Pl}}}},
$$

does not share the same mapping, i.e., $\rho_{\phi} e^{-\beta\left(\phi-\phi_{0}\right) / M_{\mathrm{P} 1}}$ in the demonimator does not equate to $\rho_{\mathrm{DE}}$ of equation (2.12). Thus, even if the $\beta^{2}$ term should be absent in equation (3.5), the evolution of $\delta_{\mathrm{DM}}$ and hence $\Phi_{H}$ in the chameleon model cannot be mapped to that in a noninteracting scenario with a dark energy equation of state $w_{\text {eff }}$. In other words, the evolution of $\delta_{\mathrm{DM}}$ and $\Phi_{H}$ in the low-redshift universe probes a combination of $\left(\alpha, h, \omega_{\mathrm{DM}}\right)$ different from that determined by $w_{\text {eff }}$.

Note that while measurements of the CMB angular damping scale $\theta_{\mathrm{d}}=r_{\mathrm{d}}^{*} / D_{\mathrm{A}}^{*}$ by $\mathrm{ACT}$ [31] and SPT [32] have been instrumental in procuring the first CMB-only constraints 
on the radiation energy density, for the chameleon model $\theta_{\mathrm{d}}$ offers no new information beyond what can already be obtained from the acoustic peaks. To see this, we note that the diffusion damping scale $r_{\mathrm{d}}^{*} \equiv r_{\mathrm{d}}\left(a_{*}\right)$ can be approximated as [40]

$$
\begin{aligned}
r_{\mathrm{d}}^{* 2} & \approx(2 \pi)^{2} \int_{0}^{a_{*}} \frac{d a}{a^{3} \sigma_{T} n_{e} H}\left[\frac{R^{2}+(16 / 15)(1+R)}{6\left(1+R^{2}\right)}\right] \\
& \propto \frac{(2 \pi)^{2}}{\sqrt{\omega_{\mathrm{DM}} e^{-\alpha\left(h^{2} / \omega_{\mathrm{DM}}-1\right)}+\omega_{\mathrm{B}}}} \int_{0}^{a_{*}} \frac{d a}{a \sqrt{a+a_{\mathrm{eq}}} \sigma_{T} n_{e}}\left[\frac{R^{2}+(16 / 15)(1+R)}{6\left(1+R^{2}\right)}\right],
\end{aligned}
$$

where $\sigma_{T}$ is the Thomson scattering cross-section, and $n_{e}$ is the free electron number density. Clearly, after $z_{\text {eq }}$ and $\omega_{\mathrm{B}}$ have been fixed, $r_{\mathrm{d}}^{*}$ is likewise completely determined, while the angular diameter distance $D_{\mathrm{A}}^{*}$ is probed by the angular sound horizon scale $\theta_{\mathrm{s}}$. The information contained in the angular diffusion scale $\theta_{\mathrm{d}}$ is therefore redundant. Indeed, as we shall see in section 4, adding ACT and SPT data to WMAP's acoustic peak measurements does not improve the constraints on the chameleon model parameters.

\subsection{Non-CMB observables}

One more orthogonal parameter combination needs to be constrained in order to eliminate all degeneracies. This can come from any one of the following observables:

1. The Hubble expansion parameter $h$ can be fixed directly by measurements in our local neighbourhood.

2. The current generation of baryon acoustic oscillations (BAO) measurements constrains the angular scale $r_{\mathrm{s}}\left(z_{\mathrm{d}}\right) / D_{\mathrm{V}}\left(z_{\mathrm{BAO}}\right)$, where $z_{\mathrm{d}} \approx 1020$ denotes the redshift of the baryon drag epoch, $z_{\mathrm{BAO}}$ the effective redshift at which the BAO is observed, and

$$
D_{\mathrm{V}}(z)=\left[z(1+z)^{2} \frac{D_{\mathrm{A}}^{2}(z)}{H(z)}\right]^{1 / 3}
$$

is a distance scale subsuming both the angular diameter distance $D_{\mathrm{A}}(z)$ and the Hubble expansion rate

$$
H(a) \propto \sqrt{\left(\omega_{\mathrm{DM}}+\omega_{\mathrm{B}}\right) a^{-3}+\left(h^{2}-\omega_{\mathrm{DM}}-\omega_{\mathrm{B}}\right) e^{-3 \int_{1}^{a} d \ln a^{\prime}\left(1+w_{\mathrm{eff}}\right)}}, .
$$

The BAO peak shares a common physical origin with the CMB acoustic peaks. However, because the BAO measurement takes place at a low redshift $\left(z_{\mathrm{BAO}}<1\right)$, the parameter combination to which it is sensitive is vastly different from that probed by the high-redshift $r_{\mathrm{s}}^{*} / D_{\mathrm{A}}^{*}$ of equations (3.2) and (3.3).

3. An alternative low-redshift distance constraint comes from measurements of the luminosity distance $D_{\mathrm{L}}(a)$ versus redshift using type Ia supernovae. In a flat spatial geometry, $D_{\mathrm{L}}(a)=D_{\mathrm{A}}(a) / a^{2}$.

4. The large-scale matter power spectrum extracted from galaxy clustering surveys yields several useful pieces of information. Firstly, the "turning point" depends on the comoving wavenumber

$$
\left.k_{\mathrm{eq}} \equiv a_{\mathrm{eq}} H\left(a_{\mathrm{eq}}\right) \approx 4.7 \times 10^{-4} h^{-1} \sqrt{\left(\omega_{\mathrm{DM}} e^{-\alpha\left(h^{2} / \omega_{\mathrm{DM}}-1\right)}+\omega_{\mathrm{B}}\right)\left(1+z_{\mathrm{eq}}\right.}\right) h \mathrm{Mpc}^{-1},
$$


which, after fixing $z_{\mathrm{eq}}$ and $\omega_{\mathrm{B}}$ with the CMB, effectively depends only on the Hubble parameter $h$ (recall that $k$ is measured in units of $h \mathrm{Mpc}^{-1}$ ). Secondly, BAO features are also present in the present generation of power spectrum measurements (e.g., [45]), which offers the same handle on a low-redshift distance scale as discussed immediately above. Thirdly, the fifth force induced by the DM-DE coupling in principle enhances clustering in a scale-dependent fashion even on scales well within the Hubble horizon according to equation (3.5), thereby distorting the matter power spectrum. However, because the range of the force has only reached Hubble length at recent times, we expect this subhorizon spectral distortion to be confined mainly to the nonlinear scales $\left(k \gtrsim 0.2 \mathrm{hMpc}^{-1}\right)$, where the density perturbations have had more time to evolve under the influence of the fifth force [29] and which are beyond the scope of our perturbative analysis. Nonetheless, we note that in certain $f(R)$ or interacting quintessence models it is possible to shift the clustering enhancement to the linear scales (e.g., [46, 47]).

Other low-redshift matter distribution measurements such as the cluster mass function [48-50], cosmic shear [51-53], and the Lyman- $\alpha$ forest [54, 55] are also potentially useful for constraining chameleon cosmology in that they provide a direct measurement of the density fluctuation amplitude which should be sensitive to the non-standard low-redshift evolution of the matter density perturbations induced by the DM-DE coupling. However, to make use of these measurements requires nonlinear modelling, which is beyond the scope of this work.

\section{Cosmological data analysis}

Following the discussions of section 3, we consider four generic types of cosmological measurements: temperature and polar polarisation power spectra of the CMB anisotropies, the BAO scale, the large-scale matter power spectrum, and direct measurements of the local Hubble expansion rate. The details of the actual data sets used are related below. On these data sets we perform a Bayesian statistical inference analysis using the publicly available Markov Chain Monte Carlo (MCMC) parameter estimation package CosmoMC [56] coupled to the CAMB [42] Boltzmann solver modified in accordance with section 2. With the exception of the local Hubble parameter measurement, the likelihood routines and the associated window functions are supplied by the experimental collaborations.

Table 1 shows the fit parameters used in the analysis and their associated (flat) priors ranges. We adopt the Gelman-Rubin convergence criterion $R-1<0.02$ when generating our multiple Markov chains, where $R$ is the variance of chain means divided by the mean of chain variances.

\subsection{Data sets}

We split our analysis into two stages, the first centred on the WMAP 9-year measurements of the cosmic microwave background temperature and polarisation anisotropies [57], and the second on the more recent temperature measurements from the first data release of the Planck mission [5]. We describe these and other auxiliary data sets below.

CMB anisotropies. In the stage 1, we use the temperature (TT), E-polarisation (EE), and $B$-polarisation (BB) autocorrelation power spectra, as well as the temperature- $E$-polarisation (TE) cross-correlation power spectra from WMAP [5]. To this we add the TT power spectra from ACT and SPT [58-62], which cover the range $600<\ell<3000$ (SPT) and $500<$ $\ell<10000$ (ACT). The ACT/SPT data include in addition the deflection power spectra 


\begin{tabular}{|c|c|c|c|}
\hline Parameter & Description & Prior & $\overline{\Lambda \mathrm{CDM}}$ \\
\hline$\omega_{\mathrm{B}}$ & Baryon density & $0.005 \rightarrow 0.04$ & - \\
\hline$\omega_{\mathrm{DM}}$ & Cold dark matter density & $0.01 \rightarrow 0.99$ & - \\
\hline$h$ & Hubble parameter & $0.4 \rightarrow 1.0$ & - \\
\hline$z_{\mathrm{re}}$ & Redshift of reionization & $3 \rightarrow 35$ & - \\
\hline$n_{\mathrm{S}}$ & Spectral index of the primordial power spectrum & $0.5 \rightarrow 1.5$ & - \\
\hline $\ln \left(10^{10} A_{\mathrm{S}}\right)$ & Amplitude of the primordial power spectrum at $k=0.05 \mathrm{Mpc}^{-1}$ & $2.7 \rightarrow 4.0$ & - \\
\hline$\alpha$ & Slope of the chameleon potential & $10^{-5} \rightarrow 5$ & 0 \\
\hline$\beta$ & Strength of the chameleon coupling & $10^{-5} \rightarrow 5$ & 0 \\
\hline$N_{\text {eff }}$ & Number of effective massless neutrino families & $1 \rightarrow 9.8$ & 3.046 \\
\hline
\end{tabular}

Table 1. Fitting parameters in our MCMC analysis and their associated flat prior ranges. The six parameters in the first block are the standard $\Lambda$ CDM parameters. The chameleon model parameter space subsumes the $\Lambda$ CDM parameter space, and has an additional two parameters $\alpha$ and $\beta$ which, when set to zero, reduce the chameleon model to $\Lambda$ CDM. The last entry $N_{\text {eff }}$ parameterises the radiation excess; in $\Lambda \mathrm{CDM}$ this is fixed at 3.046 .

$(\phi \phi)$ due to gravitational lensing, which we also analyse in our study. In stage 2 , following the guidelines of [5], we use the TT spectra from the Planck mission in conjunction with the WMAP 9-year polarisation measurements (WP). To this we add the ACT/SPT TT spectrum at high multipoles (HighL), which helps to constrain the foreground nuisance parameters used in the Planck data analysis.

Local Hubble parameter measurements. We adopt the values of $H_{0}$ measured in our local neighbourhood by the Hubble Space Telescope. In stage 1, we use $H_{0}=74.2 \pm 3.6 \mathrm{~km}$ $\mathrm{s}^{-1} \mathrm{Mpc}^{-1}$ [63], published in 2009, in conjunction with our analysis of the WMAP 9-year CMB measurements. This value of $H_{0}$ was also adopted by the WMAP collaboration in their 9-year analysis [57], and we opt to keep it to facilitate comparison. In stage 2, we adopt a more recent measured value, $H_{0}=73.8 \pm 2.4 \mathrm{~km} \mathrm{~s}^{-1} \mathrm{Mpc}^{-1}$ [64], in combination with our Planck analysis. Because each value is used exclusively with either WMAP or Planck, we adopt the same acronym HST for both measurements.

Large-scale matter power spectrum We use the red luminous galaxy survey from the Sloan Digital Sky Survey DR-7 (SDSS) [45] to constrain the matter power spectrum in the $k$-range $[0.02,0.2] h \mathrm{Mpc}^{-1}$, in conjunction with the WMAP 9-year data in stage 1 of the analysis. The power spectrum measurement contains both broadband information on the scale-dependence, as well as geometric information in the form wiggles from the baryonic acoustic oscillations. In combination with the WMAP CMB measurements, the latter information generally suffices to constrain model parameters that affect the late-time expansion of the universe, while the former is primarily useful for extended models in which the perturbations pick up an additional scale-dependence at late times (e.g., models with finite neutrino masses) [65]. In the context of chameleon models, we expect geometric information to be the more useful of the two (the additional scale-dependence in chameleon models are in any case all confined to the nonlinear scales beyond $k \sim 0.2 \mathrm{~h} \mathrm{Mpc}^{-1}$, as discussed in section 3). We therefore do not use the matter power spectrum in stage 2 of the analysis in combination with Planck data, and opt instead for direct measurements of the BAO scale. See next.

BAO scale. We use measurements of the BAO scale by SDSS-DR [66], SDSS-DR9 [67], and 6dFGS [68]. For cosmological models with no non-trivial scale dependence, geometric 


\begin{tabular}{|c|c|c|c|c|c|c|c|c|}
\hline Model & Data & $\alpha$ & $\beta$ & $N_{\text {eff }}$ & $\omega_{\mathrm{DM}}$ & $h$ & $\Delta \chi_{\mathrm{a}}^{2} / \Delta_{\text {dof }}$ & $\Delta \chi_{\mathrm{b}}^{2} / \Delta_{\text {dof }}$ \\
\hline \multirow{10}{*}{ 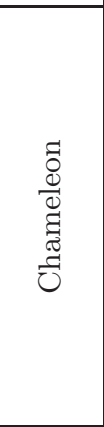 } & $\mathrm{W}+\mathrm{HST}$ & $<0.23$ & $<0.19$ & 3.046 & $0.109_{0.099}^{0.118}$ & $0.70_{0.65}^{0.75}$ & $+0.0 / 2$ & $+0.1 / 1$ \\
\hline & $\mathrm{W}+\mathrm{HST}+\mathrm{ACT} / \mathrm{SPT}$ & $<0.24$ & $<0.19$ & 3.046 & $0.109_{0.100}^{0.119}$ & $0.70_{0.65}^{0.75}$ & $+1.3 / 2$ & $+0.7 / 1$ \\
\hline & $\mathrm{W}+\mathrm{SDSS}$ & $<0.48$ & $<0.19$ & 3.046 & $0.112_{0.103}^{0.121}$ & $0.65_{0.60}^{0.70}$ & $-0.1 / 2$ & $-0.3 / 1$ \\
\hline & $\mathrm{W}+\mathrm{HST}+\mathrm{SDSS}$ & $<0.27$ & $<0.19$ & 3.046 & $0.112_{0.104}^{0.120}$ & $0.68_{0.64}^{0.71}$ & $+0.1 / 2$ & $+1.1 / 1$ \\
\hline & $\mathrm{W}+\mathrm{SNIa}+\mathrm{SDSS}$ & $<0.19$ & $<0.19$ & 3.046 & $0.113_{0.105}^{0.120}$ & $0.68_{0.65}^{0.71}$ & $-0.4 / 2$ & $-0.3 / 1$ \\
\hline & $\mathrm{P}+\mathrm{WP}+\mathrm{BAO}$ & $<0.17$ & $<0.19$ & 3.046 & $0.118_{0.115}^{0.122}$ & $0.67_{0.64}^{0.69}$ & $+0.7 / 2$ & $+0.5 / 1$ \\
\hline & $\mathrm{P}+\mathrm{WP}+\mathrm{HST}$ & $<0.09$ & $<0.19$ & 3.046 & $0.117_{0.113}^{0.122}$ & $0.68_{0.65}^{0.71}$ & $+0.4 / 2$ & $+4.7 / 1$ \\
\hline & $\mathrm{P}+\mathrm{WP}+$ HighL+BAO & $<0.17$ & $<0.19$ & 3.046 & $0.118_{0.115}^{0.122}$ & $0.67_{0.64}^{0.69}$ & $-0.7 / 2$ & $+0.4 / 1$ \\
\hline & $\mathrm{P}+\mathrm{WP}+$ HighL + HST & $<0.09$ & $<0.19$ & 3.046 & $0.117_{0.113}^{0.122}$ & $0.67_{0.64}^{0.69}$ & $-0.6 / 2$ & $+4.7 / 1$ \\
\hline & $\mathrm{P}+\mathrm{WP}+$ HighL+BAO+HST & $<0.08$ & $<0.19$ & 3.046 & $0.117_{0.114}^{0.121}$ & $0.67_{0.65}^{0.69}$ & $-0.6 / 2$ & $+3.1 / 1$ \\
\hline \multirow{10}{*}{ 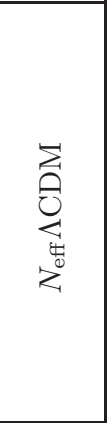 } & $\mathrm{W}+\mathrm{HST}$ & - & - & $4.3_{2.8}^{6.1}$ & $0.13_{0.10}^{0.18}$ & $0.75_{0.69}^{0.82}$ & $-0.1 / 1$ & - \\
\hline & $\mathrm{W}+\mathrm{HST}+\mathrm{ACT} / \mathrm{SPT}$ & - & - & $3.2_{2.6}^{3.9}$ & $0.14_{0.11}^{0.17}$ & $0.74_{0.69}^{0.79}$ & $+0.6 / 1$ & - \\
\hline & $\mathrm{W}+\mathrm{SDSS}$ & - & - & $4.5_{2.1}^{7.6}$ & $0.14_{0.10}^{0.21}$ & $0.75_{0.62}^{0.91}$ & $+0.2 / 1$ & - \\
\hline & $\mathrm{W}+\mathrm{HST}+\mathrm{SDSS}$ & - & - & $4.3_{3.1}^{5.5}$ & $0.14_{0.11}^{0.16}$ & $0.74_{0.68}^{0.80}$ & $-1.0 / 1$ & - \\
\hline & $\mathrm{W}+\mathrm{SNIa}+\mathrm{SDSS}$ & - & - & $4.6_{2.4}^{7.4}$ & $0.14_{0.10}^{0.20}$ & $0.76_{0.64}^{0.92}$ & $-0.1 / 1$ & - \\
\hline & $\mathrm{P}+\mathrm{WP}+\mathrm{BAO}$ & - & - & $3.3_{2.8}^{3.9}$ & $0.124_{0.114}^{0.134}$ & $0.69_{0.66}^{0.73}$ & $+0.2 / 1$ & - \\
\hline & $\mathrm{P}+\mathrm{WP}+\mathrm{HST}$ & - & - & $3.7_{3.2}^{4.2}$ & $0.128_{0.118}^{0.137}$ & $0.73_{0.69}^{0.77}$ & $-4.3 / 1$ & - \\
\hline & $\mathrm{P}+\mathrm{WP}+$ HighL $+\mathrm{BAO}$ & - & - & $3.3_{2.7}^{3.8}$ & $0.123_{0.114}^{0.132}$ & $0.65_{0.62}^{0.69}$ & $-1.1 / 1$ & - \\
\hline & $\mathrm{P}+\mathrm{WP}+$ HighL+HST & - & - & $3.2_{2.7}^{3.6}$ & $0.119_{0.111}^{0.128}$ & $0.68_{0.65}^{0.72}$ & $-5.3 / 1$ & - \\
\hline & $\mathrm{P}+\mathrm{WP}+$ HighL+BAO+HST & - & - & $3.2_{2.7}^{3.6}$ & $0.120_{0.112}^{0.128}$ & $0.68_{0.66}^{0.71}$ & $-3.8 / 1$ & - \\
\hline
\end{tabular}

Table 2. 1D marginal statistics for the chameleon parameters $\alpha$ and $\beta$ in the chameleon fit (top block) and for $N_{\text {eff }}$ in the $N_{\text {eff }} \Lambda$ CDM fit (bottom block) derived from various data combinations. The abbreviation "W" stands for WMAP, while "P" is Planck. For one-sided intervals we show only the $95 \%$ credible limit, while for two-sided intervals we show the $95 \%$ central credible interval (see [70] for definition) as well as the posterior mean. The second last column compares the model's best-fit $\chi^{2}$ value relative to the $\Lambda \mathrm{CDM}$ best-fit as per equation (4.1) in the face of $\Delta_{\text {dof }}$ additional degrees of freedom, while the last column contrasts the chameleon and the $N_{\text {eff }} \Lambda \mathrm{CDM}$ fits as per equation (4.2).

information extracted from large-scale structure surveys suffices to constrain the model parameters [65], and has the added advantage of being much less prone to the nonlinearity issues that plague broadband measurements of the large-scale matter power spectrum.

Type Ia supernovae. We include the supernovae data set Union2 compilation [69], labelled "SNIa", in stage 1 of the analysis. The standardised luminosity of Type Ia supernovae is a classic measure of the redshift-dependence of the ( $h$-normalised) luminosity distance. This compilation consists of 557 supernovae collected from different surveys, and was a standard compilation used also by the WMAP collaboration in their 9-year data analysis [57].

\subsection{Parameter constraints}

Table 2 summarises the constraints on the chameleon model parameters $\alpha$ and $\beta$ from our MCMC analysis using various data combinations. The corresponding two-dimensional marginal posteriors in the $(\alpha, \beta)$-plane are displayed in figure 3.

Clearly, none of the data combinations prefer the chameleon model over the basic $\Lambda$ CDM model. This can be discerned firstly from the fact that only one-sided (instead of two-sided) limits exist for the chameleon parameters $\alpha$ and $\beta$, indicating that $\alpha=\beta=0$ are completely compatible with all data combinations. ${ }^{3}$ Secondly, the different between the best-fit $\chi^{2}$ values

\footnotetext{
${ }^{3}$ Note that in practice, because we use equations (2.15) and (2.16) to determine the present-day field
} 

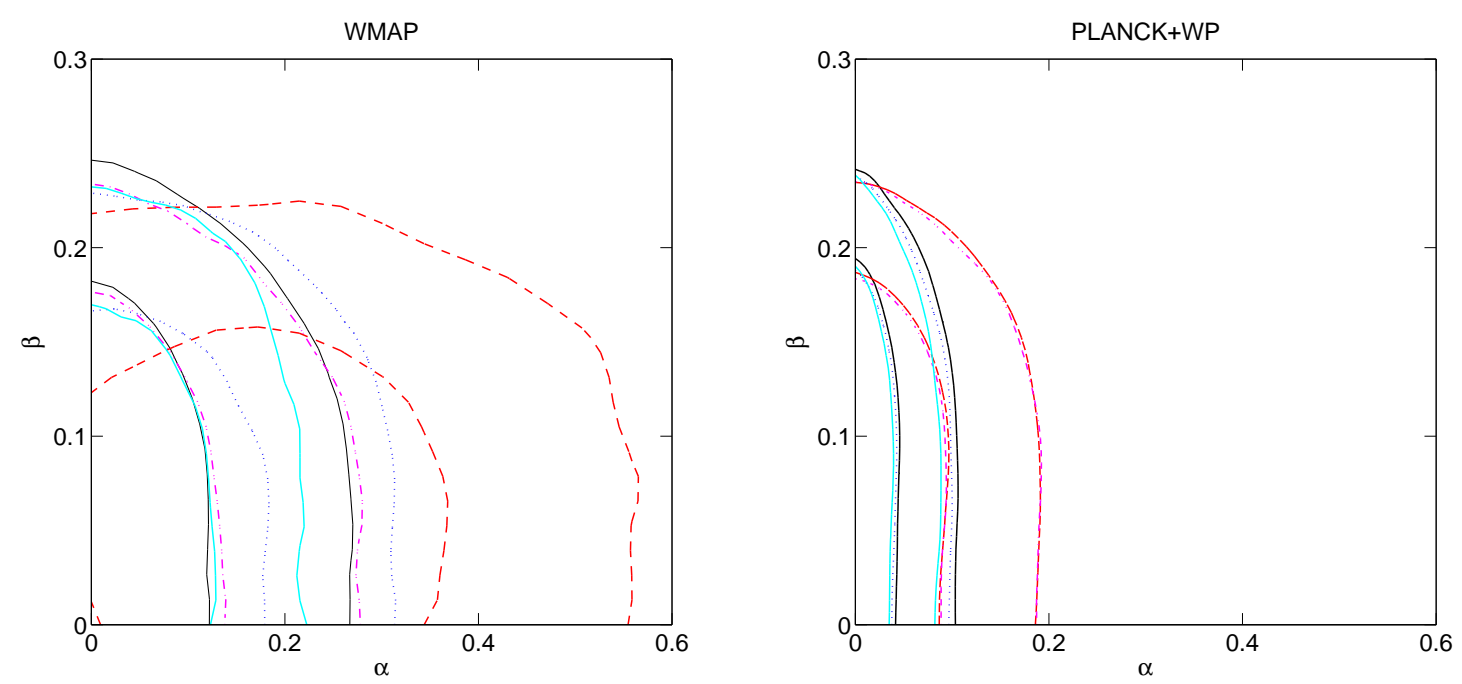

Figure 3. 2D marginal $68 \%$ and $95 \%$ contours in the $(\alpha, \beta)$-plane derived from various data combinations. Left: WMAP temperature and polarisation measurements combined with HST (solid black), SDSS (dashed red), HST+SDSS (dotted blue), HST+ACT/SPT (dot-dashed magenta), and SDSS+SNIa (solid cyan). Right: Planck temperature and WMAP polarisation data combined with HST (solid black), BAO (dashed red), HighL+HST (dotted blue), HighL+BAO (dot-dashed magenta), and HighL+HST+BAO (solid cyan).

of the chameleon and the $\Lambda \mathrm{CDM}$ model,

$$
\Delta \chi_{\mathrm{a}}^{2}=\chi_{\text {model }}^{2}-\chi_{\Lambda \mathrm{CDM}}^{2}
$$

is at best -0.66 for the Planck $+\mathrm{WP}+$ HighL $+\mathrm{BAO}$ data combination. This is barely beyond the reliability limit of $\left|\Delta \chi^{2}\right| \geq 0.6$ of the BOBYQA bounded minimisation routine [71] used to find the best-fit $\chi^{2}$ values (the Metropolis-Hastings algorithm in standard MCMC analyses is not suitable for finding the maximum of a multi-dimensional likelihood function), and is in any case too small an improvement to the fit to warrant the introduction of two extra fit parameters.

In terms of the limits on the chameleon parameters $\alpha$ and $\beta$, there is virtually no difference between data combinations in their constraining power on the coupling parameter $\beta$; all return the same $95 \%$ upper limit $\beta<0.19$. This can be understood as follows: as already discussed in sections 2.2 and 3.1, neither the effective dark energy equation of state $w_{\text {eff }}$ nor the high-redshift observable $z_{\mathrm{eq}}$ is sensitive to $\beta$; the effect of $\beta$ is felt only through the late ISW effect, because of the enhanced Bardeen potential $\Phi_{H}$ at $z \lesssim 1$ on close to the Hubble length as shown in figure 2. Figure 4 shows the CMB TT spectrum of various chameleon models relative to the $\Lambda \mathrm{CDM}$ best-fit to the Planck+WP+HST data combination. Although the $\beta$-dependence is clearly strong, the late ISW effect evidently manifests itself only at low multipoles $\ell \lesssim 30$. This means firstly that, besides the CMB TT spectrum, the cosmological observations considered in this analysis play no role in constraining $\beta$. Secondly, swapping WMAP for Planck CMB temperature data, both of which are limited only by cosmic variance

value $\phi_{0}$ and the field mass $M_{\phi}$, it is necessary to impose a nonzero lower limit on the priors on $\alpha$ and $\beta$, both chosen here to be $10^{-5}$ as shown in table 1 , in order to avoid an artificial divergence at $\alpha=\beta=0$. However, because the phenomenology of $\alpha=\beta=10^{-5}$ is for all purposes indistinguishable from $\Lambda \mathrm{CDM}$, we choose not to enforce the distinction between the two cases. 


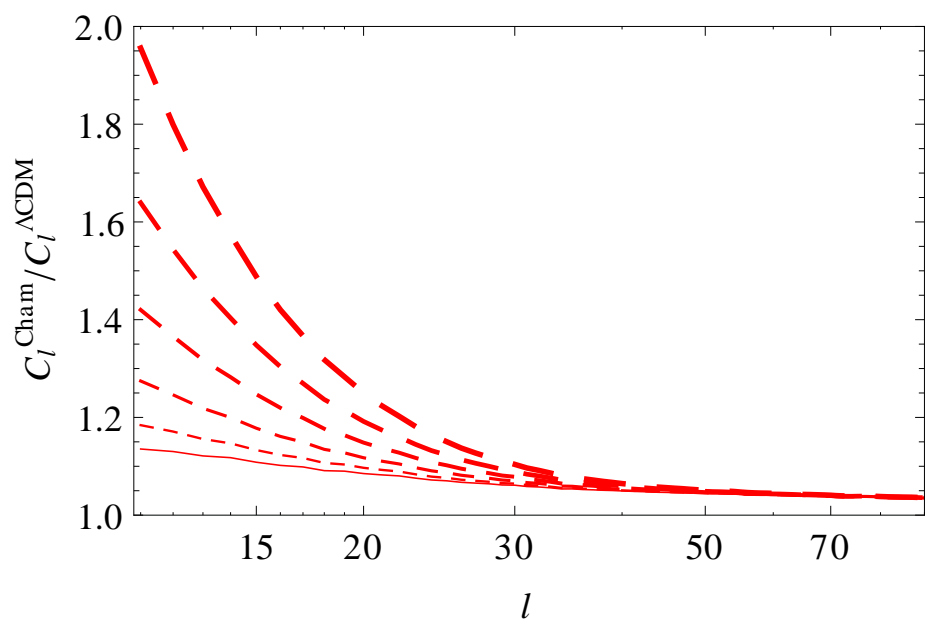

Figure 4. CMB TT spectrum of various chameleon models relative to the $\Lambda$ CDM case. The $\Lambda$ CDM spectrum corresponds to the best-fit model of the data set Planck $+\mathrm{WP}+\mathrm{HST}$, while the chameleon models have the common parameters $\left(\alpha, \omega_{\mathrm{DM}}, h\right)=(0.1,0.117,0.688)$ and, from bottom to top, $\beta=$ $0.05,0.1,0.15,0.2,0.25,0.3$.

at the low multipoles, also cannot improve the sensitivity to $\beta$. Further improvements can come about by either looking at the effects of the fifth force induced by $\beta$ on very nonlinear scales, or, in the future, the impact of the $\beta$-dependent metric perturbations on full-sky cosmic shear surveys such as the ESA Euclid mission [72]

For the slope parameter $\alpha$, the constraint generally improves when WMAP data are swapped for Planck measurements. This can be understood as a consequence both of a more well-constrained $z_{\mathrm{eq}}$ and of a more accurate determination of $\theta_{\mathrm{s}}$. The latter in particular constrains the effective equation of state $w_{\text {eff }}$, whose departure from the canonical value of -1 is now, as shown in figure 5 and table 3 , restricted to no more than $20 \%$ (at $95 \%$ confidence) at $z<0.5$ and $50 \%$ at $z<0.8$. Adding the HST measurement of $H_{0}$ to Planck improves the constraint on $\alpha$ even further (almost a factor of two). However, this improvement may be an artefact originating from the $2 \sigma+$ discrepancy between the HST measurement and the $h$ value inferred from Planck data in the minimal $\Lambda$ CDM model $[5,6]$. Indeed, keeping $\omega_{\mathrm{DM}}$ fixed, we see, on the one hand, from equation (3.1) that $\alpha$ must decrease with an increasing $h$ in order to maintain the same $z_{\text {eq }}$. On the other hand, from equation (3.3), maintaining the same $D_{\mathrm{A}}^{*}$ in the face of a smaller $\alpha$ requires that we reduce $h$. Thus, the net effect is that $\alpha$ goes down, while the inferred value of $h$ changes only marginally, as suggested by the numbers in table 2 . This incompatibility between data sets is also confirmed by the $2 \mathrm{D}$ marginal posterior in the $\left(\alpha, H_{0}\right)$-plane in figure 6 , which shows a clear preference for a negative $\alpha$ value - which is not within the chameleon parameter space - if $H_{0}$ was indeed as large as $73.8 \mathrm{~km} \mathrm{~s}^{-1} \mathrm{Mpc}^{-1}$ preferred by HST.

Comparing our results with previous investigations of interacting DM-DE models, our limits differ in significant ways. The analyses of $[29,73]$ dealt with a scenario identical to ours, and it was argued in these works that $\beta \sim \mathcal{O}(1)$ could be compatible with observations. However, this conclusion was based on the argument that with $\beta$ and $\alpha$ fixed at 1 and 0.2 respectively, a best-fit $\chi^{2}$ "close to" the $\Lambda$ CDM best-fit could be obtained when the usual $\Lambda \mathrm{CDM}$ parameters are let to vary freely. Our analysis here, in contrast, allows all parameters to vary, so as to locate the values of $\alpha$ and $\beta$ actually preferred by the observational data. 

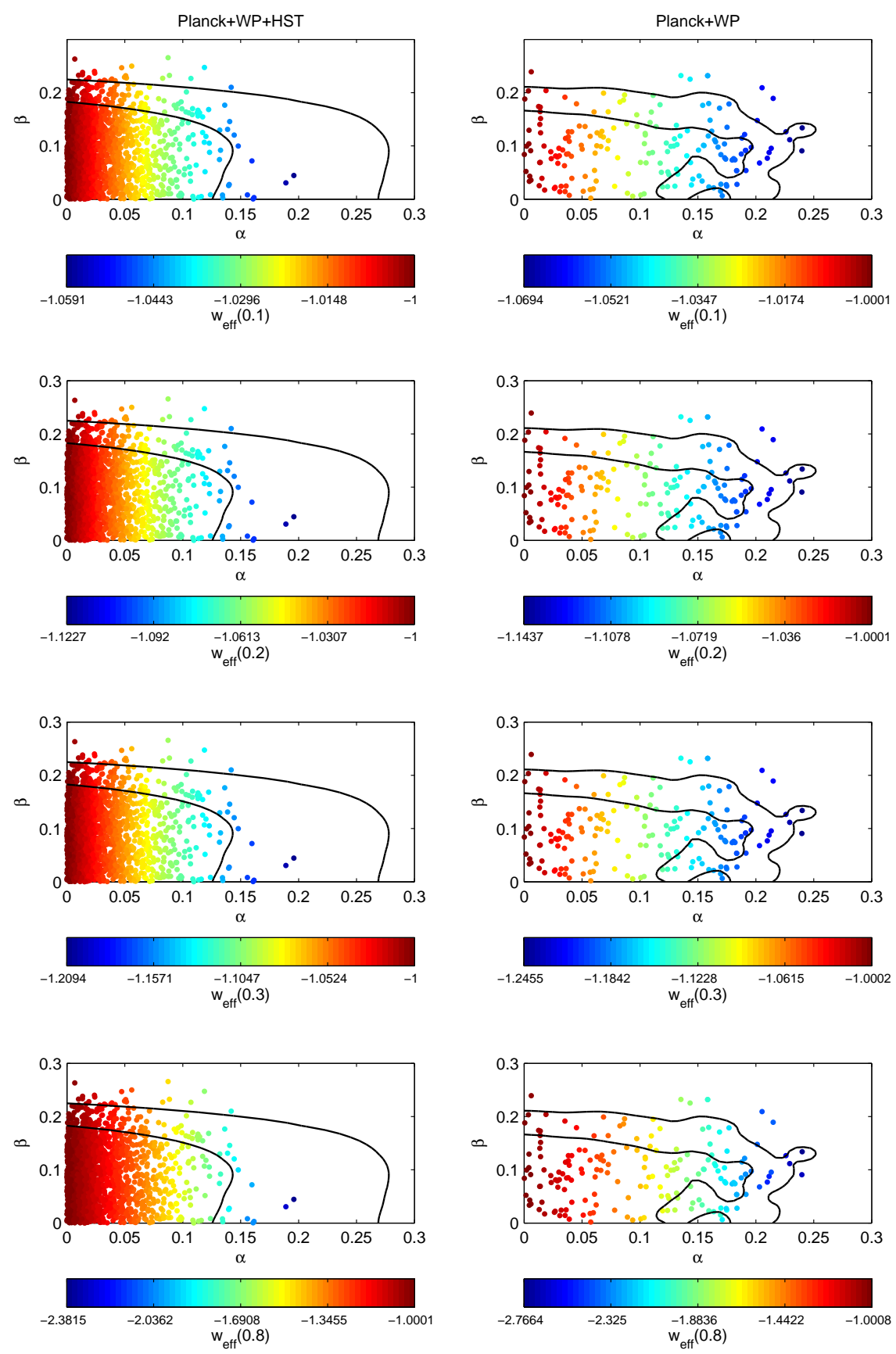

Figure 5. 2D marginal $68 \%$ and $95 \%$ contours in the $(\alpha, \beta)$-plane derived from Planck+WP+HST (left) and Planck+WP (right), and the corresponding effective equation of state for the dark energy (coloured dots) at four different redshifts, from top to bottom, $z=0.1,0.2,0.3,0.8$.

References [74] and [75] also considered a coupled scenario in which the scalar potential $V(\phi)$ is of the runaway form (2.8), and obtained constraints on $\beta$ that are considerably tighter than ours. We note however that the coupling function adopted in these analyses is of the form $f(\phi)=e^{-\beta \phi / M_{\mathrm{Pl}}}$, which differs from ours by a crucial minus sign, signifying that their effective potential $V_{\text {eff }}(\phi)$ does not possess a local minimum, nor $\phi(t)$ an adiabatic attractor 


\begin{tabular}{|c|c|c|}
\hline \multirow{2}{*}{$z$} & \multicolumn{2}{|c|}{$\mathrm{w}_{\mathrm{eff}}(z)$} \\
\cline { 2 - 3 } & Planck+WP+HST & Planck+WP \\
\hline 0.0 & $>-1.003$ & $>-1.005$ \\
\hline 0.1 & $>-1.03$ & $>-1.06$ \\
\hline 0.2 & $>-1.06$ & $>-1.12$ \\
\hline 0.3 & $>-1.11$ & $>-1.21$ \\
\hline 0.4 & $>-1.16$ & $>-1.32$ \\
\hline 0.5 & $>-1.2$ & $>-1.5$ \\
\hline 0.6 & $>-1.3$ & $>-1.7$ \\
\hline 0.7 & $>-1.4$ & $>-2.0$ \\
\hline 0.8 & $>-1.5$ & $>-2.4$ \\
\hline
\end{tabular}

Table 3. 1D marginal $95 \%$ lower limit for the effective dark energy equation of state $w_{\text {eff }}$ in the chameleon model at various redshifts, derived from the data combinations Planck $+\mathrm{WP}+\mathrm{HST}$ and Planck+WP.

solution. For this reason, the results of [74] and [75] cannot be meaningfully compared with ours in the context of parameter inference.

\subsection{Chameleon mimicking dark radiation?}

As discussed at the beginning of section 3, the nonstandard time-evolution induced for the dark matter density by the DM-DE coupling tends to delay the epoch of matter-radiation equality relative to the standard $\Lambda \mathrm{CDM}$ case with the same $\omega_{\mathrm{DM}}$. This raises the possibility that chameleon phenomenology might mimic the effect of dark radiation, which also has shifting $z_{\text {eff }}$ as its primary effect. We test this possibility by fitting the $\Lambda$ CDM model extended with a free $N_{\text {eff }}$ to the same data combinations explored in section 4.2 for the chameleon model, and compute in each case the constraints on $N_{\text {eff }}$ as well as the best-fit $\chi^{2}$ value relative to $\Lambda \mathrm{CDM}$ as defined in equation (4.1). In addition, for each data combination we evaluate the $\chi^{2}$ difference between the best-fit chameleon model and its $N_{\text {eff }} \Lambda \mathrm{CDM}$ counterpart, defined as

$$
\Delta \chi_{\mathrm{b}}^{2}=\chi_{\text {chameleon }}^{2}-\chi_{N_{\text {eff }} \Lambda \mathrm{CDM}}^{2} .
$$

This $\Delta \chi_{\mathrm{b}}^{2}$ value will tell us whether or not chameleon models are able to mimic dark radiation.

Table 2 shows the results of this exercise. Note that in deriving these constraints we have made use of the BBN consistency relation [76] in CosmoMC, which automatically adjusts the helium mass fraction $Y_{\mathrm{He} 4}$ according to big bang nucleosynthesis predictions for each set of input parameters $N_{\text {eff }}$ and $\omega_{\mathrm{B}}$. Clearly, only the combinations WMAP+HST+SDSS and Planck $+\mathrm{WP}+\mathrm{HST}$ show a $2 \sigma+$ preference for $N_{\text {eff }}>3.046$, and in the latter case, this is accompanied by a dramatic increase in the inferred $h$ value $\left(h=0.73_{0.69}^{0.77}\right)$ relative to other Planck data combinations. In terms of the best-fit $\chi^{2}$, we find $\Delta \chi_{\mathrm{a}}^{2}$ values of -0.98 and -4.32 respectively relative to $\Lambda \mathrm{CDM}$, suggesting a mild preference for a radiation excess especially in the latter case.

Comparing the chameleon model with $N_{\text {eff }} \Lambda \mathrm{CDM}$ for these same two data combinations, we find the chameleon scenario consistently the worse performer of the two. The bestfit $\chi^{2}$ differences between the two models as per definition $(4.2)$ are +1.13 and +4.68 for $\mathrm{WMAP}+\mathrm{HST}+\mathrm{SDSS}$ and Planck $+\mathrm{WP}+\mathrm{HST}$ respectively. This can be traced to the fact that phenomenologically, the $\alpha$ parameter needs to be negative in order to accommodate the large $H_{0}$ value preferred by HST (see figure 6 and discussion in section 4.2). A negative $\alpha$, however, 


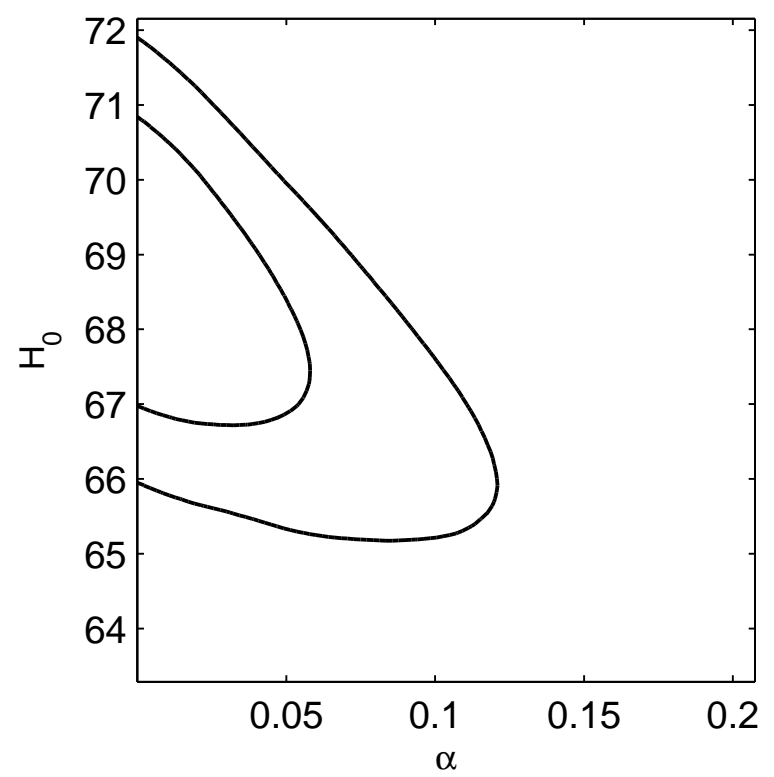

Figure 6. $2 \mathrm{D}$ marginal posterior in the $\left(\alpha, H_{0}\right)$-plane derived from Planck $+\mathrm{WP}+\mathrm{HST}$.

does not belong to the chameleon parameter space. Thus, despite a passing similarity at the equality epoch, the degeneracy between $H_{0}$ and the chameleon model parameter $\alpha$ ultimately works in the wrong direction to be able to resolve the tension between the HST measurement and the Planck-inferred $H_{0}$ value.

At this point, we note that our chameleon model is distinct from early dark energy models which $d o$ mimic a radiation excess because the dark energy equation of state in these models evolves to $\sim 1 / 3$ during radiation domination [77]. In contrast, the attractor solution in chameleon models ensures that the scalar field remains slowly rolling while sitting in the effective minimum of the potential, and therefore maintains a cosmological constant-like behaviour at all times.

\section{$5 \quad$ Future tests}

The two main predictions of the chameleon model are the apparent phantom behaviour of the effective dark energy equation of state $w_{\text {eff }}$ and a fifth force that enhances the clustering of dark matter as well as the gravitational potentials. The former is, for any nonzero $\beta$, solely a function of the parameter $\alpha$, while the latter is strongly dependent on $\beta$.

As we have seen in figure 5 and table 3 , the effective dark energy equation of state is already constrained at $95 \%$ confidence to $w_{\text {eff }}>-1.2$ at $z=0.5$ and $<-1.5$ at $z=0.8$ by Planck $+\mathrm{WP}+\mathrm{HST}$. It would therefore appear at first glance that the Dark Energy Survey (DES), with $1 \sigma$ sensitivities $\sigma\left(w_{0}\right) \sim 0.078$ and $\sigma\left(w_{a}\right) \sim 0.3[78]$ for the parameterisation

$$
w(a)=w_{0}+w_{a}(1-a),
$$

may not be sufficient to further tighten the constraint on the chameleon parameter $\alpha$. The ESA Euclid mission on the other hand, with $\sigma\left(w_{0}\right) \sim 0.018$ and $\sigma\left(w_{a}\right) \sim 0.15$ [72], should produce some improvements.

We caution however that this interpretation is not strictly correct: parameter sensitivities derived for a simple dynamical dark model such as equation (5.1) do not automatically 
translate to bounds on the chameleon model parameters. This is because ongoing and future large-scale structure surveys will derive their constraints on the dark energy equation of state from both distance measures and the growth of density perturbations; apart from its effect on the late ISW effect, the latter observable has not been used in the present analysis of existing cosmological data. Recall from our discussions in section 3.1 that in the chameleon scenario, the evolution of the dark matter density parameter $\Omega_{\mathrm{DM}}(t)$, equation (3.7), which governs the gravitational potential felt by the density perturbations, maps to an effective dark energy equation of state different from the canonical $w_{\text {eff }}$ responsible for the background Hubble expansion. This additional feature should allow us to distinguish cleanly between simple dynamical dark energy and interacting/chameleon models. A detailed analysis of how well future surveys will be able to select between dark energy models is beyond the scope of the present work, but we note that publicly available forecast codes such as [79-81] exist which, with minor modifications, will be equal to the task.

As for the fifth force, there are two possible places to look for its effects. One is the very nonlinear scales, where the perturbations have had the most time to evolve under the force's influence. Given that the $\beta$ parameter is already constrained by the late ISW effect to less than 0.2 , we expect the fifth force to manifest itself on subhorizon scales, according to equation (3.5), as a maximum $8 \%$ correction to the (scale-dependent) effective Newtonian constant; nonlinear evolution should further enhance the effect (although difficult to predict precisely). The second possibility is to take advantage of the almost full sky coverage of future surveys such as the ESA Euclid mission. In the same way that the scale-dependent evolution of the Bardeen potential $\Phi_{H}$ at $z \lesssim 1$ and $k \sim(a H)^{-1}$ induced by $\beta$ (see figure 2) enhances the late ISW effect, we expect an analogous distortion of, e.g., the cosmic shear convergence angular power spectrum, at similarly low multipoles $\ell \lesssim 30$. Of course, detection of this signature and ultimately the extent to which one could improve upon existing constraints on $\beta$ will still be, like the late ISW effect, limited by cosmic variance. To address this question quantitatively we would need to perform a parameter sensitivity forecast, which we defer to a future work.

\section{Conclusions}

We have considered in this work a simple interacting DM-DE/chameleon model in which a scalar field slowly rolls and adiabatically tracks the minimum of an effective potential starting from deep in the radiation-dominated era. We have tested this model against recent cosmological observations, especially new CMB temperature measurements from the Planck mission, and have found constraints on the two model parameters: $\alpha$, slope of the scalar potential, and $\beta$, the coupling strength. We find that while the constraint on the coupling strength $\beta$ has remained unchanged between WMAP and Planck, essentially because this parameter is constrained primarily by the late ISW effect, the upper limit on $\alpha$, which has the dual effect of shifting the matter-radiation equality epoch and altering the effective dark energy equation of state, has improved somewhat.

Because of its potential to shift the epoch of matter-radiation equality, we have tested also the possibility that such a chameleon model might mimic the phenomenology of dark radiation, and resolve the apparent tension between the relatively large Hubble expansion rate measured in our local neighbourhood by HST and the low value inferred from the Planck CMB data. We find however that the degeneracy between $\alpha$ and $H_{0}$ in the chameleon model works in the wrong direction: a larger $H_{0}$ value in tends to push $\alpha$ towards zero, i.e., the 
cosmological constant limit. In fact, the ultimate effect of the HST/Planck tension on the chameleon model is to put an artificially tight constraint on $\alpha$.

Taking into account the allowed model parameter space, we have discussed possible signatures of this type of chameleon model for ongoing and future large-scale structure surveys such as DES [78] and the ESA Euclid mission [72]. The salient effects are (i) a mismatch between the effective dark energy equation of state inferred from the background expansion and from the growth function which should allow us to determine or further constrain the value of $\alpha$, and (ii) distortion of the large-scale distribution power spectrum on very nonlinear and close-to-horizon scales, both of which are predominantly sensitive to $\beta$. To determine the precise sensitivities of DES or Euclid to the chameleon model parameters would however require a dedicated forecast analysis. We leave this exercise for a future work.

\section{Acknowledgments}

DB thanks the Institut für Theoretische Teilchenphysik und Kosmologie at RWTH Aachen for their hospitality during the development of this work. DB acknowledges financial support from CAPES-PDEE (grant 6864-10-4), and the use of computing resources at CENAPADSP/UNICAMP and CCJDR-IFGW/UNICAMP.

\section{References}

[1] Supernova Search Team Collaboration, A. G. Riess et al., "Observational evidence from supernovae for an accelerating universe and a cosmological constant," Astron.J. 116 (1998) 1009-1038, arXiv:astro-ph/9805201 [astro-ph].

[2] Supernova Cosmology Project Collaboration, S. Perlmutter et al., "Measurements of Omega and Lambda from 42 high redshift supernovae," Astrophys.J. 517 (1999) 565-586, arXiv:astro-ph/9812133 [astro-ph].

[3] N. Suzuki, D. Rubin, C. Lidman, G. Aldering, R. Amanullah, et al., "The Hubble Space Telescope Cluster Supernova Survey: V. Improving the Dark Energy Constraints Above zi1 and Building an Early-Type-Hosted Supernova Sample," Astrophys.J. 746 (2012) 85, arXiv:1105.3470 [astro-ph.CO].

[4] B. D. Sherwin, J. Dunkley, S. Das, J. W. Appel, J. R. Bond, et al., "Evidence for dark energy from the cosmic microwave background alone using the Atacama Cosmology Telescope lensing measurements," Phys.Rev.Lett. 107 (2011) 021302, arXiv:1105.0419 [astro-ph.CO].

[5] Planck Collaboration, P. Ade et al., "Planck 2013 results. XVI. Cosmological parameters," arXiv: 1303.5076 [astro-ph.CO].

[6] Planck Collaboration, P. Ade et al., "Planck 2015 results. XIII. Cosmological parameters," arXiv:1502.01589 [astro-ph.CO].

[7] C. Wetterich, "Cosmology and the Fate of Dilatation Symmetry," Nucl.Phys. B302 (1988) 668.

[8] B. Ratra and P. Peebles, "Cosmological Consequences of a Rolling Homogeneous Scalar Field," Phys.Rev. D37 (1988) 3406.

[9] R. Caldwell, R. Dave, and P. J. Steinhardt, "Cosmological imprint of an energy component with general equation of state," Phys.Rev.Lett. 80 (1998) 1582-1585, arXiv:astro-ph/9708069 [astro-ph].

[10] L. Lombriser, A. Slosar, U. Seljak, and W. Hu, "Constraints on $\mathrm{f}(\mathrm{R})$ gravity from probing the large-scale structure," Phys.Rev. D85 (2012) 124038, arXiv:1003.3009 [astro-ph.C0]. 
[11] J. Dossett, B. Hu, and D. Parkinson, "Constraining models of $f(R)$ gravity with Planck and WiggleZ power spectrum data," JCAP 1403 (2014) 046, arXiv:1401.3980 [astro-ph.C0].

[12] J. Bel, P. Brax, C. Marinoni, and P. Valageas, "Cosmological tests of modified gravity: constraints on $F(R)$ theories from the galaxy clustering ratio,"

arXiv: 1406.3347 [astro-ph.CO].

[13] L. Amendola, "Coupled quintessence," Phys.Rev. D62 (2000) 043511, arXiv:astro-ph/9908023 [astro-ph].

[14] T. Faulkner, M. Tegmark, E. F. Bunn, and Y. Mao, "Constraining f(R) Gravity as a Scalar Tensor Theory," Phys.Rev. D76 (2007) 063505, arXiv:astro-ph/0612569 [astro-ph].

[15] W. Hu and I. Sawicki, "A Parameterized Post-Friedmann Framework for Modified Gravity," Phys.Rev. D76 (2007) 104043, arXiv:0708.1190 [astro-ph].

[16] E. Bertschinger and P. Zukin, "Distinguishing Modified Gravity from Dark Energy," Phys.Rev. D78 (2008) 024015, arXiv:0801.2431 [astro-ph].

[17] L. P. Chimento, A. S. Jakubi, D. Pavon, and W. Zimdahl, "Interacting quintessence solution to the coincidence problem," Phys.Rev. D67 (2003) 083513, arXiv:astro-ph/0303145 [astro-ph].

[18] D. Comelli, M. Pietroni, and A. Riotto, "Dark energy and dark matter," Phys.Lett. B571 (2003) 115-120, arXiv:hep-ph/0302080 [hep-ph].

[19] C. M. Will, "The Confrontation between general relativity and experiment," Living Rev.Rel. 4 (2001) 4, arXiv:gr-qc/0103036 [gr-qc].

[20] D. Kapner, T. Cook, E. Adelberger, J. Gundlach, B. R. Heckel, et al., "Tests of the gravitational inverse-square law below the dark-energy length scale," Phys.Rev.Lett. 98 (2007) 021101, arXiv:hep-ph/0611184 [hep-ph].

[21] C. Hoyle, D. Kapner, B. R. Heckel, E. Adelberger, J. Gundlach, et al., "Sub-millimeter tests of the gravitational inverse-square law," Phys.Rev. D70 (2004) 042004, arXiv:hep-ph/0405262 [hep-ph].

[22] S.-Q. Yang, B.-F. Zhan, Q.-L. Wang, C.-G. Shao, L.-C. Tu, et al., "Test of the Gravitational Inverse Square Law at Millimeter Ranges," Phys.Rev.Lett. 108 (2012) 081101.

[23] J. Khoury and A. Weltman, "Chameleon fields: Awaiting surprises for tests of gravity in space," Phys.Rev.Lett. 93 (2004) 171104, arXiv:astro-ph/0309300 [astro-ph].

[24] J. Khoury and A. Weltman, "Chameleon cosmology," Phys.Rev. D69 (2004) 044026, arXiv:astro-ph/0309411 [astro-ph].

[25] P. Brax, C. van de Bruck, A.-C. Davis, J. Khoury, and A. Weltman, "Detecting dark energy in orbit - The Cosmological chameleon," Phys.Rev. D70 (2004) 123518, arXiv: astro-ph/0408415 [astro-ph].

[26] P. Brax, C. van de Bruck, A.-C. Davis, and A. M. Green, "Small scale structure formation in chameleon cosmology," Phys.Lett. B633 (2006) 441-446, arXiv:astro-ph/0509878 [astro-ph].

[27] E. J. Copeland, M. Sami, and S. Tsujikawa, "Dynamics of dark energy," Int.J.Mod.Phys. D15 (2006) 1753-1936, arXiv:hep-th/0603057 [hep-th].

[28] J. Wang, L. Hui, and J. Khoury, "No-Go Theorems for Generalized Chameleon Field Theories," Physical Review Letters 109 (2012) no. 24, arXiv:1208.4612 [astro-ph.C0].

[29] S. Das, P. S. Corasaniti, and J. Khoury, "Super-acceleration as signature of dark sector interaction," Phys.Rev. D73 (2006) 083509, arXiv:astro-ph/0510628 [astro-ph].

[30] T. Damour and A. M. Polyakov, "The String dilaton and a least coupling principle," Nucl.Phys. B423 (1994) 532-558, arXiv:hep-th/9401069 [hep-th]. 
[31] J. Dunkley, R. Hlozek, J. Sievers, V. Acquaviva, P. Ade, et al., "The Atacama Cosmology Telescope: Cosmological Parameters from the 2008 Power Spectra," Astrophys.J. 739 (2011) 52, arXiv:1009.0866 [astro-ph.CO].

[32] R. Keisler, C. Reichardt, K. Aird, B. Benson, L. Bleem, et al., "A Measurement of the Damping Tail of the Cosmic Microwave Background Power Spectrum with the South Pole Telescope," Astrophys.J. 743 (2011) 28, arXiv:1105.3182 [astro-ph.CO].

[33] J. Hamann, S. Hannestad, G. G. Raffelt, I. Tamborra, and Y. Y. Wong, "Cosmology seeking friendship with sterile neutrinos," Phys.Rev.Lett. 105 (2010) 181301, arXiv:1006.5276 [hep-ph].

[34] J. Hamann, S. Hannestad, G. G. Raffelt, and Y. Y. Wong, "Sterile neutrinos with eV masses in cosmology: How disfavoured exactly?," JCAP 1109 (2011) 034, arXiv:1108.4136 [astro-ph.CO].

[35] H. Vogel and J. Redondo, "Dark Radiation constraints on minicharged particles in models with a hidden photon," JCAP 1402 (2014) 029, arXiv:1311.2600 [hep-ph].

[36] J. Hasenkamp, "Daughters mimic sterile neutrinos (almost!) perfectly," arXiv:1405.6736 [astro-ph.CO].

[37] O. E. Bjaelde and S. Das, "Dark Matter Decaying into a Fermi Sea of Neutrinos," Phys.Rev. D82 (2010) 043504, arXiv:1002.1306 [astro-ph.CO].

[38] P. Di Bari, S. F. King, and A. Merle, "Dark Radiation or Warm Dark Matter from long lived particle decays in the light of Planck," Phys.Lett. B724 (2013) 77-83, arXiv:1303.6267 [hep-ph].

[39] E. V. Linder and G. Robbers, "Shifting the Universe: Early Dark Energy and Standard Rulers," JCAP 0806 (2008) 004, arXiv:0803.2877 [astro-ph].

[40] Z. Hou, R. Keisler, L. Knox, M. Millea, and C. Reichardt, "How Massless Neutrinos Affect the Cosmic Microwave Background Damping Tail," Phys.Rev. D87 (2013) 083008, arXiv:1104.2333 [astro-ph.CO].

[41] M. Archidiacono, S. Hannestad, A. Mirizzi, G. Raffelt, and Y. Y. Wong, "Axion hot dark matter bounds after Planck," JCAP 1310 (2013) 020, arXiv:1307.0615 [astro-ph.C0].

[42] A. Lewis, A. Challinor, and A. Lasenby, "Efficient computation of CMB anisotropies in closed FRW models," Astrophys.J. 538 (2000) 473-476, arXiv:astro-ph/9911177 [astro-ph].

[43] R. Bean, E. E. Flanagan, and M. Trodden, "Adiabatic instability in coupled dark energy-dark matter models," Phys.Rev. D78 (2008) 023009, arXiv:0709.1128 [astro-ph].

[44] P. S. Corasaniti, "Slow-Roll Suppression of Adiabatic Instabilities in Coupled Scalar Field-Dark Matter Models," Phys.Rev. D78 (2008) 083538, arXiv:0808.1646 [astro-ph].

[45] B. A. Reid, W. J. Percival, D. J. Eisenstein, L. Verde, D. N. Spergel, et al., "Cosmological Constraints from the Clustering of the Sloan Digital Sky Survey DR7 Luminous Red Galaxies," Mon.Not.Roy.Astron.Soc. 404 (2010) 60-85, arXiv:0907.1659 [astro-ph.CO].

[46] H. Motohashi, A. A. Starobinsky, and J. Yokoyama, "Cosmology Based on f(R) Gravity Admits 1 eV Sterile Neutrinos," Phys.Rev.Lett. 110 (2013) no. 12, 121302, arXiv:1203.6828 [astro-ph.CO].

[47] P. Brax and A.-C. Davis, "Modified Gravity and the CMB," Phys.Rev. D85 (2012) 023513, arXiv:1109.5862 [astro-ph.CO].

[48] C. Reichardt, B. Stalder, L. Bleem, T. Montroy, K. Aird, et al., "Galaxy clusters discovered via the Sunyaev-Zel'dovich effect in the first 720 square degrees of the South Pole Telescope survey," Astrophys.J. 763 (2013) 127, arXiv:1203.5775 [astro-ph.C0]. 
[49] Planck Collaboration, P. Ade et al., "Planck 2013 results. XXIX. Planck catalogue of Sunyaev-Zeldovich sources," Astron.Astrophys. (2013), arXiv:1303.5089 [astro-ph.C0].

[50] A. B. Mantz, A. von der Linden, S. W. Allen, D. E. Applegate, P. L. Kelly, et al., "Weighing the Giants IV: Cosmology and Neutrino Mass," arXiv:1407.4516 [astro-ph.CO].

[51] T. Schrabback, J. Hartlap, B. Joachimi, M. Kilbinger, P. Simon, et al., "Evidence for the accelerated expansion of the Universe from weak lensing tomography with COSMOS," Astron.Astrophys. 516 (2010) A63, arXiv:0911.0053 [astro-ph.C0].

[52] M. J. Jee, J. A. Tyson, M. D. Schneider, D. Wittman, S. Schmidt, et al., "Cosmic shear results from the deep lens survey - I: Joint constraints on $\omega_{m}$ and $\sigma_{8}$ with a two-dimensional analysis," Astrophys.J. 765 (2013) 74, arXiv:1210.2732 [astro-ph.CO].

[53] CFHTLenS Collaboration, T. Kitching et al., "3D Cosmic Shear: Cosmology from CFHTLenS," arXiv:1401.6842 [astro-ph.CO].

[54] SDSS Collaboration, P. McDonald et al., "The Lyman-alpha forest power spectrum from the Sloan Digital Sky Survey," Astrophys.J.Suppl. 163 (2006) 80-109, arXiv:astro-ph/0405013 [astro-ph].

[55] K.-G. Lee, S. Bailey, L. E. Bartsch, W. Carithers, K. S. Dawson, et al., "The BOSS Lyman-alpha Forest Sample from SDSS Data Release 9," arXiv:1211.5146 [astro-ph.C0].

[56] A. Lewis and S. Bridle, "Cosmological parameters from CMB and other data: A Monte Carlo approach," Phys.Rev. D66 (2002) 103511, arXiv:astro-ph/0205436 [astro-ph].

[57] WMAP Collaboration, G. Hinshaw et al., "Nine-Year Wilkinson Microwave Anisotropy Probe (WMAP) Observations: Cosmological Parameter Results," Astrophys.J.Suppl. 208 (2013) 19, arXiv:1212.5226 [astro-ph.CO].

[58] J. Dunkley, E. Calabrese, J. Sievers, G. Addison, N. Battaglia, et al., "The Atacama Cosmology Telescope: likelihood for small-scale CMB data," arXiv:1301.0776 [astro-ph.CO].

[59] S. Das, T. Louis, M. R. Nolta, G. E. Addison, E. S. Battistelli, et al., "The Atacama Cosmology Telescope: temperature and gravitational lensing power spectrum measurements from three seasons of data," JCAP 1404 (2014) 014, arXiv:1301.1037 [astro-ph.CO].

[60] K. Story, C. Reichardt, Z. Hou, R. Keisler, K. Aird, et al., "A Measurement of the Cosmic Microwave Background Damping Tail from the 2500-square-degree SPT-SZ survey," Astrophys.J. 779 (2013) 86, arXiv:1210.7231 [astro-ph.CO].

[61] Z. Hou, C. Reichardt, K. Story, B. Follin, R. Keisler, et al., "Constraints on Cosmology from the Cosmic Microwave Background Power Spectrum of the 2500-square degree SPT-SZ Survey," Astrophys.J. 782 (2014) 74, arXiv:1212.6267 [astro-ph.CO].

[62] E. Calabrese, R. A. Hlozek, N. Battaglia, E. S. Battistelli, J. R. Bond, et al., "Cosmological parameters from pre-planck cosmic microwave background measurements," Phys.Rev. D87 (2013) no. 10, 103012, arXiv:1302.1841 [astro-ph.C0].

[63] A. G. Riess, L. Macri, S. Casertano, M. Sosey, H. Lampeitl, et al., "A Redetermination of the Hubble Constant with the Hubble Space Telescope from a Differential Distance Ladder," Astrophys.J. 699 (2009) 539-563, arXiv:0905.0695 [astro-ph.CO].

[64] A. G. Riess, L. Macri, S. Casertano, H. Lampeitl, H. C. Ferguson, A. V. Filippenko, S. W. Jha, W. Li, and R. Chornock, "A 3\% Solution: Determination of the Hubble Constant with the Hubble Space Telescope and Wide Field Camera 3," APJ 730 (2011), arXiv:1103.2976 [astro-ph.CO].

[65] J. Hamann, S. Hannestad, J. Lesgourgues, C. Rampf, and Y. Y. Y. Wong, "Cosmological parameters from large scale structure - geometric versus shape information," JCAP 7 (2010) 22, arXiv:1003.3999 [astro-ph.CO]. 
[66] N. Padmanabhan, X. Xu, D. J. Eisenstein, R. Scalzo, A. J. Cuesta, et al., "A 2 per cent distance to $z=0.35$ by reconstructing baryon acoustic oscillations - I. Methods and application to the Sloan Digital Sky Survey," Mon.Not.Roy.Astron.Soc. 427 (2012) no. 3, 2132-2145, arXiv: 1202.0090 [astro-ph.CO].

[67] L. Anderson, E. Aubourg, S. Bailey, D. Bizyaev, M. Blanton, et al., "The clustering of galaxies in the SDSS-III Baryon Oscillation Spectroscopic Survey: Baryon Acoustic Oscillations in the Data Release 9 Spectroscopic Galaxy Sample," Mon.Not.Roy.Astron.Soc. 427 (2013) no. 4, 3435-3467, arXiv:1203.6594 [astro-ph.C0].

[68] F. Beutler, C. Blake, M. Colless, D. H. Jones, L. Staveley-Smith, et al., "The 6dF Galaxy Survey: Baryon Acoustic Oscillations and the Local Hubble Constant," Mon.Not.Roy.Astron.Soc. 416 (2011) 3017-3032, arXiv:1106.3366 [astro-ph.C0].

[69] R. Amanullah, C. Lidman, D. Rubin, G. Aldering, P. Astier, K. Barbary, M. S. Burns, A. Conley, K. S. Dawson, S. E. Deustua, M. Doi, S. Fabbro, L. Faccioli, H. K. Fakhouri, G. Folatelli, A. S. Fruchter, H. Furusawa, G. Garavini, G. Goldhaber, A. Goobar, D. E. Groom, I. Hook, D. A. Howell, N. Kashikawa, A. G. Kim, R. A. Knop, M. Kowalski, E. Linder, J. Meyers, T. Morokuma, S. Nobili, J. Nordin, P. E. Nugent, L. Östman, R. Pain, N. Panagia, S. Perlmutter, J. Raux, P. Ruiz-Lapuente, A. L. Spadafora, M. Strovink, N. Suzuki, L. Wang, W. M. Wood-Vasey, N. Yasuda, and T. Supernova Cosmology Project, "Spectra and Hubble Space Telescope Light Curves of Six Type Ia Supernovae at $0.511<\mathrm{z}<1.12$ and the Union2 Compilation," ApJ 716 (2010), arXiv:1004.1711 [astro-ph.CO].

[70] J. Hamann, S. Hannestad, G. Raffelt, and Y. Y. Wong, "Observational bounds on the cosmic radiation density," JCAP 0708 (2007) 021, arXiv:0705.0440 [astro-ph].

[71] M. J. D. Powell, "The BOBYQA algorithm for bound constrained optimization without derivatives," DAMTP 2009/NA06 .

[72] A. Refregier, A. Amara, T. Kitching, A. Rassat, R. Scaramella, et al., "Euclid Imaging Consortium Science Book," arXiv:1001.0061 [astro-ph.IM].

[73] P. S. Corasaniti, "The innocuousness of adiabatic instabilities in coupled scalar field-dark matter models," AIP Conf.Proc. 1241 (2010) 797-803, arXiv:1001.2687 [astro-ph.C0].

[74] G. La Vacca, J. Kristiansen, L. Colombo, R. Mainini, and S. Bonometto, "Do WMAP data favor neutrino mass and a coupling between Cold Dark Matter and Dark Energy?," JCAP 0904 (2009) 007, arXiv:0902.2711 [astro-ph.CO].

[75] V. Pettorino, "Testing modified gravity with Planck: the case of coupled dark energy," Phys.Rev. D88 (2013) no. 6, 063519, arXiv:1305.7457 [astro-ph.C0].

[76] J. Hamann, J. Lesgourgues, and G. Mangano, "Using BBN in cosmological parameter extraction from CMB: A Forecast for PLANCK," JCAP 0803 (2008) 004, arXiv:0712.2826 [astro-ph].

[77] E. Calabrese, D. Huterer, E. V. Linder, A. Melchiorri, and L. Pagano, "Limits on Dark Radiation, Early Dark Energy, and Relativistic Degrees of Freedom," Phys.Rev. D83 (2011) 123504, arXiv:1103.4132 [astro-ph.Co].

[78] A. Albrecht, G. Bernstein, R. Cahn, W. L. Freedman, J. Hewitt, et al., "Report of the Dark Energy Task Force," arXiv:astro-ph/0609591 [astro-ph].

[79] J. Hamann, S. Hannestad, and Y. Y. Wong, "Measuring neutrino masses with a future galaxy survey," JCAP 1211 (2012) 052, arXiv:1209.1043 [astro-ph.CO].

[80] T. Basse, O. E. Bjaelde, J. Hamann, S. Hannestad, and Y. Y. Wong, "Dark energy properties from large future galaxy surveys," JCAP 1405 (2014) 021, arXiv:1304.2321 [astro-ph.C0].

[81] T. Basse, J. Hamann, S. Hannestad, and Y. Y. Y. Wong, "Getting leverage on inflation with a large photometric redshift survey," arXiv:1409.3469 [astro-ph.C0]. 